\title{
Optimal control of unsteady compressible viscous flows
}

\author{
S. Scott Collis ${ }^{1, *, \dagger}$, Kaveh Ghayour ${ }^{2, \ddagger}$, Matthias Heinkenschloss ${ }^{3, \S}$, \\ Michael Ulbrich ${ }^{4, \Phi}$ and Stefan Ulbrich ${ }^{5, \|}$ \\ ${ }^{1}$ Department of Mechanical Engineering and Materials Science, Rice University, Houston, \\ TX 77005-1892, U.S.A. \\ ${ }^{2}$ Department of Computational and Applied Mathematics and Department of Mechanical Engineering and \\ Materials Science, Rice University, Houston, TX 77005-1892, U.S.A. \\ ${ }^{3}$ Department of Computational and Applied Mathematics, Rice University, Houston, TX 77005-1892, U.S.A. \\ ${ }^{4}$ Fachbereich Mathematik, Universität Hamburg, Bundesstr. 55, D-20146 Hamburg, Germany \\ ${ }^{5}$ Lehrstuhl für Angewandte Mathematik und Mathematische Statistik, Zentrum Mathematik, \\ Technische Universität München, D-80290 München, Germany
}

\begin{abstract}
SUMMARY
The control of complex, unsteady flows is a pacing technology for advances in fluid mechanics. Recently, optimal control theory has become popular as a means of predicting best case controls that can guide the design of practical flow control systems. However, most of the prior work in this area has focused on incompressible flow which precludes many of the important physical flow phenomena that must be controlled in practice including the coupling of fluid dynamics, acoustics, and heat transfer. This paper presents the formulation and numerical solution of a class of optimal boundary control problems governed by the unsteady two-dimensional compressible Navier-Stokes equations. Fundamental issues including the choice of the control space and the associated regularization term in the objective function, as well as issues in the gradient computation via the adjoint equation method are discussed. Numerical results are presented for a model problem consisting of two counter-rotating viscous vortices above an infinite wall which, due to the self-induced velocity field, propagate downward and interact with the wall. The wall boundary control is the temporal and spatial distribution of wall-normal velocity. Optimal controls for objective functions that target kinetic energy, heat transfer, and wall shear stress are presented along with the influence of control regularization for each case. Copyright (c) 2002 John Wiley \& Sons, Ltd.
\end{abstract}

KEY WORDS: optimal flow control; compressible Navier-Stokes equations; gradient computation

\footnotetext{
* Correspondence to: S. Scott Collis, Department of Mechanical Engineering and Materials Science, Rice University, Houston, TX 77005-1892, U.S.A.

† E-mail: collis@rice.edu

¥E-mail: kghayour@caam.rice.edu

$\S$ E-mail: heinken@rice.edu

TE-mail: ulbrich@math.uni-hamburg.de

IIE-mail: sulbrich@ma.tum.de
}

Contract/grant sponsor: Texas ATP; contract/grant number: 003604-001-1999

Contract/grant sponsor: Los Alamos Computer Science Institute; contract/grant number: 03891-99-23

Contract/grant sponsor: DFG; contract/grant number: UL157/3-1, UL158/2-1

Contract/grant sponsor: CRPC; contract/grant number: CCR-9120008

Contract/grant sponsor: NSF SCREMS; contract/grant number: 98-72009

Copyright (c) 2002 John Wiley \& Sons, Ltd.

Received 21 May 2002

Revised 22 July 2002 


\section{INTRODUCTION}

In this paper, we study the optimal boundary control of flows governed by the two-dimensional, unsteady, compressible Navier-Stokes equations. Our research is motivated by the potential to develop novel and effective flow control strategies for inherently compressible phenomena including aeroacoustics and heat transfer by utilizing optimal control theory. The main purpose of this paper, however, is not the study of a specific application, but the investigation of some fundamental issues arising in the numerical solution of optimal control problems for unsteady compressible flows.

There has been a significant amount of research on optimal control and optimal design for problems governed by the steady and unsteady incompressible Navier-Stokes equations as well as on the optimization of compressible steady-state Euler and Navier-Stokes equations in the context of optimal design. The papers [1-11] present a sample of recent activities in the former category and References [12-17] illustrate activities in the latter category. To the authors' knowledge, the present paper documents the first approach to the optimal boundary control of unsteady compressible viscous flows. Our research is guided by the previous work on optimal flow control and design. Because different flow control and design problems were studied, past research has only addressed some of the features (time-dependence of the governing equations, compressibility of the flow, and control inputs that are distributed over portions of the boundary and over time) present in our problems.

One goal of this paper is the investigation of the mathematical formulation of the optimal control of unsteady compressible viscous flows through suction and blowing on a portion $\Gamma_{c}$ of the boundary. For a general discussion of the role of control through suction/blowing on the boundary see Reference [18]. Specifically we investigate the selection of the space of optimal controls as well as the related choice of the regularization term in the objective function. These choices are important for the mathematical well-posedness of the optimal control problems and are therefore fundamental to a numerical solution. A detailed statement of our problem formulation is given in Section 2. For the following discussion we note that $\left(t_{0}, t_{f}\right)$ is the time interval during which we control the flow, $\Gamma_{c}$ is the controlled boundary and $g$ is the control. Different choices of the control space are discussed in Section 2.2. These choices are motivated by the existing work on control of incompressible Navier-Stokes equations. In References [1,7,11] the control is only time dependent and in Reference [10] the control varies in time and only over a small portion of the boundary, i.e. it is essentially dependent only one time. Therefore, in References $[1,7,10,11]$ the regularization term for the control involves only $\int_{t_{0}}^{t_{f}} \int_{\Gamma_{c}}|g|^{2}+\left|g_{t}\right|^{2}$. In the papers $[2,3,11]$ the control varies over a large portion of the boundary and in time, but the regularization terms used are essentially $\int_{t_{0}}^{t_{f}} \int_{\Gamma_{c}}|g|^{2}$. In view of the analytical results in References $[19,20]$ this may be insufficient. In References [19,20] boundary control problems for the 2D incompressible Navier-Stokes equations are studied and well-posedness is shown if the regularization term for the control involves $\int_{t_{0}}^{t_{f}} \int_{\Gamma_{c}}\left|g_{t}\right|^{2}+|g|^{k}, k \geqslant 3$. In order to work in control spaces that are computationally more convenient, [4] uses a regularization term that involves $\int_{t_{0}}^{t_{f}} \int_{\Gamma_{c}}\left|g_{t}\right|^{2}+|g|^{2}+|\nabla g|^{2}$. Thus, even in the case of boundary control problems for incompressible Navier-Stokes equations, the choice of the regularization term still seems to be an open issue. Our problem differs from References $[1,4,7,10,11,19,20]$ in that the system is governed by the unsteady compressible Navier-Stokes equations and that high-order finite-difference methods are used for the discretization. Hence a careful study of the choice of the control space as well as the related 
choice of the regularization term is important. In particular, our numerical studies in Section 7 suggest that in contrast to the work $[2,3,10,11,18,19]$ the regularization term needs to include temporal as well as first-order spatial derivatives. If the control is posed on a subset of the boundary, it seems necessary to include second-order spatial derivatives as well. We demonstrate numerically that if temporal or spatial derivatives of the control are not included in the regularization term, the computed controls are highly oscillatory in space or time. If temporal or spatial derivatives of the control are penalized, these oscillations disappear while not affecting the original objective significantly. This indicates that a proper choice of the regularization term avoids an artificial ill-conditioning of the problem. See also Reference [11].

The choice of the control space also enters the gradient computation. The gradient has to be computed with respect to the inner product in the control space. In most cases this requires the solution of another elliptic differential equation whose domain is $\left(t_{0}, t_{f}\right) \times \Gamma_{c}$. If higher order spatial and time discretizations are used, the solution of this equation is cumbersome and expensive. In Section 4.3 we propose a new, equivalent reformulation of the problem that avoids this task. While simple, this problem reformulation is effective and it is also applicable to other optimal control problems, such as [4], where the same issue arises.

This article complements and extends our recent conference papers [21,22]. The paper [21] presents formal computations of the objective function gradient and the corresponding adjoint equations on the partial differential equation level for the compressible Navier-Stokes equations, which is not included here. The paper [22] is a predecessor of the current paper that presents our initial formulation and early results. The current paper extends upon [22] by providing a more complete problem formulation with particular attention given to the reformulation introduced for problems involving the time-derivative of the control. In addition, the current paper supplies more extensive numerical results for the control of wall-vortex interaction - a model problem motivated by the blade-vortex interaction phenomena that commonly occurs for rotorcraft. Our numerical results include control of heat transfer, wall shear stress, and kinetic energy all using controls defined over a subset of the wall boundary. These results provide interesting insight into the physics and control of compressible flows while serving as a thorough suite of problems to both demonstrate and test the features of our optimal control formulation.

\section{PROBLEM FORMULATION}

\subsection{The compressible Navier-Stokes equations}

The spatial domain occupied by the fluid is $\Omega=\left\{\mathbf{x} \in \mathbb{R}^{2}: x_{2}>0\right\}$ and $\Gamma$ denotes its spatial boundary. The portion of the boundary on which suction and blowing is applied is the controlled boundary

$$
\Gamma_{c}=\left\{\mathbf{x}=\left(x_{1}, 0\right): x_{1} \in[a, b]\right\}
$$

Let

$$
\mathbf{u}=\left(\rho, v_{1}, v_{2}, T\right)^{\mathrm{T}}
$$


denote the primitive flow variables, where $\rho(t, \mathbf{x})$ is the density; $v_{i}(t, \mathbf{x})$ denotes the velocity in the $x_{i}$-direction, $i=1,2 ; \mathbf{v}=\left(v_{1}, v_{2}\right)^{\mathrm{T}}$; and $T(t, \mathbf{x})$ denotes the temperature. The pressure $p$ and the total energy per unit mass $E$ are given by

$$
p=\frac{\rho T}{\gamma M^{2}}, \quad E=\frac{T}{\gamma(\gamma-1) M^{2}}+\frac{1}{2} \mathbf{v}^{\mathrm{T}} \mathbf{v}
$$

respectively, where $\gamma$ is the ratio of specific heats and $M$ is the reference Mach number. We write the conserved variables $\rho, \rho v_{1}, \rho v_{2}, \rho E$ as functions of the primitive variables,

$$
\mathbf{q}(\mathbf{u})=\left(\rho, \rho v_{1}, \rho v_{2}, \rho E\right)^{\mathrm{T}}
$$

and we define the inviscid flux vectors

$$
\mathbf{F}^{1}(\mathbf{u})=\left(\begin{array}{c}
\rho v_{1} \\
\rho v_{1}^{2}+p \\
\rho v_{2} v_{1} \\
(\rho E+p) v_{1}
\end{array}\right), \quad \mathbf{F}^{2}(\mathbf{u})=\left(\begin{array}{c}
\rho v_{2} \\
\rho v_{1} v_{2} \\
\rho v_{2}^{2}+p \\
(\rho E+p) v_{2}
\end{array}\right)
$$

and the viscous flux vectors

$$
\mathbf{G}^{i}(\mathbf{u})=\frac{1}{\operatorname{Re}}\left(\begin{array}{c}
0 \\
\tau_{1 i} \\
\tau_{2 i} \\
\tau_{1 i} v_{1}+\tau_{2 i} v_{2}+\frac{\kappa}{\operatorname{Pr} M^{2}(\gamma-1)} T_{x_{i}}
\end{array}\right)
$$

$i=1,2$, where $\tau_{i j}$ are the elements of the stress tensor

$$
\tau=\mu\left(\nabla \mathbf{v}+\nabla \mathbf{v}^{\mathrm{T}}\right)+\lambda(\nabla \cdot \mathbf{v}) I
$$

Here $\mu$ and $\lambda$ are the first and second coefficients of viscosity, $\kappa$ is the thermal conductivity, $P r$ is the reference Prandtl number, and $R e$ is the reference Reynolds number. For the problems presented here, constant Prandtl number and fluid properties (viscosities and thermal conductivity) are assumed along with Stokes hypothesis for the second coefficient of viscosity, $\lambda=-2 \mu / 3$. Constant Prandtl number and fluid properties were selected to simplify the exposition and these assumptions are reasonable for the model problems considered in Section 7 which do not involve large changes in temperature. However, for general compressible flows, variable fluid properties are required and these can be easily accommodated in our formulation and will be included in future studies.

The two-dimensional compressible Navier-Stokes equations for the time interval $\left[t_{0}, t_{f}\right]$ can now be written as

$$
\mathbf{q}(\mathbf{u})_{t}+\sum_{i=1}^{2}\left(\mathbf{F}^{i}(\mathbf{u})_{x_{i}}-\mathbf{G}^{i}(\mathbf{u})_{x_{i}}\right)=\mathbf{0} \quad \text { in }\left(t_{0}, t_{f}\right) \times \Omega
$$

with boundary conditions

$$
\mathbf{B}(\mathbf{u}, g)=\mathbf{0} \quad \text { on }\left(t_{0}, t_{f}\right) \times \Gamma
$$


and initial conditions

$$
\mathbf{u}\left(t_{0}, \mathbf{x}\right)=\mathbf{u}_{0}(\mathbf{x}) \quad \text { in } \Omega
$$

The function $g$ in the boundary conditions (3) acts as the control, which, for the problems considered here, is taken to be suction and blowing in the wall normal direction on $\Gamma_{c}$, and is modelled by

$$
\mathbf{v}(t, \mathbf{x})=\mathbf{b}(t, \mathbf{x})-g(t, \mathbf{x}) \mathbf{n}(t, \mathbf{x}) \quad \text { on }\left(t_{0}, t_{f}\right) \times \Gamma_{c}
$$

Here, $\mathbf{n}$ is the unit outward normal and $\mathbf{b}$ is a given boundary velocity that satisfies the compatibility condition $\mathbf{v}_{0}(\mathbf{x})=\mathbf{b}\left(t_{0}, \mathbf{x}\right)$ for $\mathbf{x} \in \Gamma$. Due to the minus sign in (5), $g>0$ corresponds to blowing, whereas $g<0$ means that fluid is removed from the domain.

To the best of our knowledge, the question of existence and uniqueness of global solutions for the full compressible Navier-Stokes equations (2), (4) with $\Omega=\mathbb{R}^{n}, n=2,3$, is still open for large initial data. Results on the existence and uniqueness of solutions to the compressible Navier-Stokes equations may be found in Refs. [23-26]. See also the brief review in Reference [21]. Therefore, the following discussion of the control problem is only formal. However, all our numerical tests indicate that for the data we consider, a solution $\mathbf{u}=\mathbf{u}(g)$ of (2)-(4) exists.

\subsection{The optimal control problem}

Abstractly, all optimal control problems treated in this paper are of the form

$$
\min _{g \in \mathscr{G}_{\mathrm{ad}}} J(g) \stackrel{\text { def }}{=} J_{\text {obs }}(\mathbf{u}(g))+J_{\text {reg }}(g)
$$

where $g$ is the control, $\mathscr{G}_{\text {ad }}$ is the set of admissible controls, $\mathbf{u}(g)$ is the solution of the compressible Navier-Stokes equations, $J_{\mathrm{obs}}$ is the part of the cost function that represents our flow control objective (the 'observed part') and $J_{\text {reg }}(g)$ is a regularization term, typically some weighted norm of the control. The concrete choices of the set of admissible controls $\mathscr{G}_{\text {ad }}$ and of the objective function $J$ depend on the physical system we want to control (e.g. how much suction and blowing can the actuators provide, what part of the flow do we want to influence), but they also need to be chosen to ensure the existence of a solution $g_{*}$ of the optimal control problem (6).

To make the discussion concrete, we consider three specific objectives. The first objective is the minimization of kinetic energy in $\Omega_{0} \subset \Omega$ at the final time $t_{f}$,

$$
J_{\mathrm{obs}}(\mathbf{u}(g))=\frac{1}{2} \int_{\Omega_{0}} \rho\left(t_{f}, \mathbf{x}\right)\left\|\mathbf{v}\left(t_{f}, \mathbf{x}\right)\right\|_{2}^{2} \mathrm{~d} \mathbf{x}
$$

the second is the minimization of heat transfer over a subset $\Gamma_{0}$ of the bottom wall,

$$
J_{\mathrm{obs}}(\mathbf{u}(g))=\frac{1}{2} \int_{t_{0}}^{t_{f}} \int_{\Gamma_{0}} \omega(\mathbf{x})\left(\frac{\partial}{\partial \mathbf{n}} T(t, \mathbf{x})\right)^{2} \mathrm{~d} \mathbf{x}
$$


and the third objective is the minimization of shear stress over a subset $\Gamma_{0}$ of the bottom wall,

$$
J_{\mathrm{obs}}(\mathbf{u}(g))=\frac{1}{2} \int_{t_{0}}^{t_{f}} \int_{\Gamma_{0}} \omega(\mathbf{x})\left(\left(\mathbf{n}^{\perp}\right)^{\mathrm{T}} \tau(t, \mathbf{x}) \mathbf{n}\right)^{2} \mathrm{~d} \mathbf{x}
$$

In (8), (9), $\mathbf{n}=\left(n_{1}, n_{2}\right)^{\mathrm{T}}$ is the outward unit normal, $\mathbf{n}^{\perp}=\left(n_{2},-n_{1}\right)^{\mathrm{T}}$ is the tangential direction, and $\omega$ is a non-negative weighting function.

Given $J_{\text {obs }}$ we must choose $\mathscr{G}_{\text {ad }}$ and the regularization term $J_{\text {reg }}(g)$ so that (6) is wellposed. As we have indicated earlier, such results are still missing for problems governed by the compressible Navier-Stokes equations (2)-(4). Therefore, our treatment of the infinite dimensional problem is formal. It is motivated by the mathematical theory of optimal control of incompressible Navier-Stokes flow, especially by References $[4,19,20]$. In our numerical experiments, the space of controls is either

$$
\begin{gathered}
\mathscr{G}_{\mathrm{ad}}=\left\{g: g \in L^{2}\left(\left(t_{0}, t_{f}\right) ; H^{1}\left(\Gamma_{c}\right)\right), g(t, \mathbf{x})=0 \quad \text { on }\left(t_{0}, t_{f}\right) \times \partial \Gamma_{c}\right\} \\
\mathscr{G}_{\mathrm{ad}}=\left\{g: g \in L^{2}\left(\left(t_{0}, t_{f}\right) ; H^{1}\left(\Gamma_{c}\right)\right), \frac{\partial}{\partial t} g \in L^{2}\left(\left(t_{0}, t_{f}\right) ; L^{2}\left(\Gamma_{c}\right)\right),\right. \\
\left.g(t, \mathbf{x})=0 \quad \text { on }\left(t_{0}, t_{f}\right) \times \partial \Gamma_{c}, g\left(t_{0}, \mathbf{x}\right)=0 \quad \text { in } \Gamma_{c}\right\}
\end{gathered}
$$

or

$$
\begin{aligned}
\mathscr{G}_{\mathrm{ad}}= & \left\{g: g \in L^{2}\left(\left(t_{0}, t_{f}\right) ; H^{2}\left(\Gamma_{c}\right)\right), \frac{\partial}{\partial t} g \in L^{2}\left(\left(t_{0}, t_{f}\right) ; L^{2}\left(\Gamma_{c}\right)\right),\right. \\
& \left.g(t, \mathbf{x})=0, \nabla g(t, \mathbf{x})=0 \quad \text { on }\left(t_{0}, t_{f}\right) \times \partial \Gamma_{c}, g\left(t_{0}, \mathbf{x}\right)=0 \quad \text { in } \Gamma_{c}\right\}
\end{aligned}
$$

For definitions of the function spaces we refer to, e.g., References [27-29]. For the purposes of this paper it is sufficient to understand that in (11) and (12) $\nabla g$ denotes the gradient of $g$ on the boundary, which in our test problem is given by $\nabla g=g_{x_{1}}$. The first two conditions in (11) are smoothness conditions on the admissible controls in space and in time, respectively. The third condition ensures that there are no jumps in $\mathbf{b}-g \mathbf{n}$ at $\left(t_{0}, t_{f}\right) \times \partial \Gamma_{c}$, where $\partial \Gamma_{c}$ is the set of end points of $\Gamma_{c}$. The fourth condition enforces compatibility between the initial velocity field $\mathbf{v}_{0}$ and boundary data $\mathbf{b}-g \mathbf{n}$. A similar interpretation is valid for the conditions in (12) except that the required spatial regularity of the controls is one order higher. For the purposes of this paper the regularization terms $J_{\text {reg }}$ associated with these spaces are more important than the precise meaning of (11) and (12).

If our control space is (11), the regularization term $J_{\text {reg }}$ in (6) is chosen to be

$$
J_{\text {reg }}(g) \stackrel{\text { def }}{=} \int_{t_{0}}^{t_{f}} \int_{\Gamma_{c}}\left(\frac{\alpha_{1}}{2}\left\|g_{t}\right\|_{2}^{2}+\frac{\alpha_{2}}{2}\|g\|_{2}^{2}+\frac{\alpha_{3}}{2}\|\nabla g\|_{2}^{2}\right) \mathrm{d} \mathbf{x} \mathrm{d} t
$$

If our control space is (10), we use (13) with $\alpha_{1}=0$. Otherwise, if our control space is (12), the regularization term $J_{\text {reg }}$ in (6) is chosen to be

$$
J_{\text {reg }}(g) \stackrel{\text { def }}{=} \int_{t_{0}}^{t_{f}} \int_{\Gamma_{c}}\left(\frac{\alpha_{1}}{2}\left\|g_{t}\right\|_{2}^{2}+\frac{\alpha_{2}}{2}\|g\|_{2}^{2}+\frac{\alpha_{3}}{2}\|\nabla g\|_{2}^{2}+\frac{\alpha_{4}}{2}\|\Delta g\|_{2}^{2}\right) \mathrm{d} \mathbf{x} \mathrm{d} t
$$


Here $\alpha_{1}, \alpha_{2}, \alpha_{3}, \alpha_{3} \geqslant 0$ and $\Delta g$ is the Laplacian of $g$ on the boundary, in our case $\Delta g=$ $g_{x_{1} x_{1}}$. We ran experiments without regularization of the derivatives of $g$, i.e. with $\alpha_{1}=\alpha_{3}=$ $\alpha_{4}=0$ and $\alpha_{2}>0$. In these cases the optimization algorithm produced strongly oscillatory controls, which frequently led to a blow-up in the flow variables generated by our compressible Navier-Stokes solver. Therefore we use $\alpha_{2}, \alpha_{3}>0$ in all our computations reported here, only $\alpha_{1}, \alpha_{4} \geqslant 0$ are allowed to be zero. If $\alpha_{1}=0$ we do not enforce smoothness of the control in time. In particular the requirements $(\partial / \partial t) g \in L^{2}\left(\left(t_{0}, t_{f}\right) ; L^{2}\left(\Gamma_{c}\right)\right)$ and $g\left(t_{0}, \mathbf{x}\right)=0$ are dropped from (11), (12). The effect of the regularization terms on the computed controls are demonstrated in Section 7. In particular, it will be seen that the computed control can exhibit strong oscillations in time if $\alpha_{1}=0$. We mention that the choice of the control space $\mathscr{G}_{\text {ad }}$ also influences the gradient computation as shown in Section 4.

\section{SPATIAL DISCRETIZATION}

Numerical simulation of aeroacoustic phenomena demands high numerical accuracy (i.e. low dissipation and dispersion) to accurately resolve convective flow-features over a wide range of space/time scales and amplitudes. To meet this need, most prior approaches have utilized high-order accurate finite difference methods such as the compact schemes in Reference [30] and the dispersion relation preserving methods in Reference [31]. Typically these methods utilize symmetric finite-difference stencils that result in zero inherent numerical dissipation. While this is desirable for resolved scales, it is well known that unresolved scales when using high-order methods can eventually corrupt a solution and/or lead to instability if not properly controlled [32]. These error modes stem from a variety of sources including approximate boundary treatments, mesh stretching, and conflicting initial and boundary conditions. While it is desirable to prevent these errors at their source if possible, for example by improving boundary treatments, most aeroacoustic simulations utilize some form of artificial-dissipation and/or filtering to control these numerical errors. There are, of course, other methods used for aeroacoustic simulation including compact upwind differences, ENO/WENO, and discontinuous Galerkin, to name a few, and aeroacoustic simulation is an active area of research with formulations, discretizations, boundary treatments, and filtering approaches under constant development. Our study focuses on the application of optimal control to aeroacoustic problems and for this purpose, we have initially chosen to use a traditional central finite-difference method with explicit high-wavenumber damping and simple boundary treatments. Inclusion of more advanced discretizations, filtering, and boundary treatments in the context of optimal control are avenues for future research.

With this background, our compressible Navier-Stokes flow solver is based on a conservative extension of the explicit finite-difference method described in Reference [33]. The Navier-Stokes equations are formulated in a generalized co-ordinate system where the physical domain is mapped to a computational space; a unit square divided into an equally spaced grid system. This transformation allows clustering of grid points in regions of high gradients, simplifies the implementation of the boundary conditions, and allows the code to be used for moderately complex geometries. While the code supports optimized finite-differences with up to seven point stencils, for this study spatial derivatives are approximated using standard fourth-order accurate central differences in the interior with third-order biased and one-sided differences used at boundaries that are designed to enhance stability when used with explicit 
time advancement methods (see Reference [34]). The metrics associated with the generalized co-ordinate transformation are computed using the same finite difference stencils to ensure discrete conservation. To suppress the growth of high-wavenumber error modes, a fourthorder artificial dissipation term acting on the numerical fluxes is added to the right-hand side of the discretized equations. This dissipation term is computed using fourth-order accurate finite-differences and the dissipation parameter, $\varepsilon$, is chosen to damp out the error modes while avoiding excessive dissipation in the resolved scales as established through numerical experimentation. Riemann extrapolation is used on far-field boundaries as an approximate nonreflecting boundary condition. Wall boundary conditions are imposed directly on the velocity components and temperature with density computed using a one-sided approximation of the continuity equation. We reiterate, that while this approach is adequate for the optimal control problems considered here, incorporating higher-order discretizations and boundary treatments is an important area of future research.

\section{THE SEMI-DISCRETE PROBLEM}

\subsection{Problem description}

We consider the optimal control problem (6) with state equations (2) and (3) after a discretization in space has been performed. The semi-discrete states $\mathbf{u}$ and controls $g$ are vector valued functions with

$$
\mathbf{u}:\left[t_{0}, t_{f}\right] \rightarrow \mathbb{R}^{n_{u}}, \quad g:\left[t_{0}, t_{f}\right] \rightarrow \mathbb{R}^{n_{g}}
$$

We do not introduce different notation, such as superscript $h$ to denote the semi-discretized states, controls, etc. The meaning of these variables and functions should be clear from the context. We assume that the boundary conditions $g(t, x)=0, x \in \partial \Gamma_{c}$, and, if (12) is used, $\nabla g(t, x)=0, x \in \partial \Gamma_{c}$, on the control are incorporated into the spatial discretization. The state $\mathbf{u}$ is the solution of the semi-discretized Navier-Stokes equations

$$
\begin{aligned}
\mathbf{q}(\mathbf{u}(t))_{t}+\mathbf{N}(\mathbf{u}(t), g(t), t) & =0, \quad t \in\left[t_{0}, t_{f}\right] \\
\mathbf{u}\left(t_{0}\right) & =\mathbf{u}_{0}
\end{aligned}
$$

The function $\mathbf{q}: \mathbb{R}^{n_{u}} \rightarrow \mathbb{R}^{n_{u}}$ represents the discretized conserved variables and $\mathbf{N}: \mathbb{R}^{n_{u}} \times \mathbb{R}^{n_{g}} \times \mathbb{R}$ $\rightarrow \mathbb{R}^{n_{u}}$ represents the inviscid and viscid flux terms as well as boundary conditions.

We consider objective functions of the form

$$
J(g)=l^{f}\left(\mathbf{u}\left(t_{f}\right)\right)+\int_{t_{0}}^{t_{f}} l(\mathbf{u}(t), g(t)) \mathrm{d} t+\int_{t_{0}}^{t_{f}} \frac{\alpha_{1}}{2} g_{t}(t)^{\mathrm{T}} \mathbf{L}_{1} g_{t}(t)+\frac{\alpha_{2}}{2} g(t)^{\mathrm{T}} \mathbf{L}_{2} g(t) \mathrm{d} t
$$

Thus, we allow the possibility that the objective function depends on the value of the flow variables at the final time and on the flow variables distributed in time. The final time contribution to the objective is denoted by $l^{f}\left(\mathbf{u}\left(t_{f}\right)\right)$ and the distributed contribution is denoted by $\int_{t_{0}}^{t_{f}} l(\mathbf{u}(t), g(t)) \mathrm{d} t$. This enables us to treat objective functions of the type (7) and (8), (9) simultaneously. In the objective function (17),

$$
l^{f}: \mathbb{R}^{n_{u}} \rightarrow \mathbb{R}, \quad l: \mathbb{R}^{n_{u}} \times \mathbb{R}^{n_{g}} \rightarrow \mathbb{R}
$$


and $\mathbf{L}_{1}, \mathbf{L}_{2} \in \mathbb{R}^{n_{g} \times n_{g}}$ are symmetric matrices. The matrix $\mathbf{L}_{1}$ is such that $g_{t}(t)^{\mathrm{T}} \mathbf{L}_{1} g_{t}(t)$ is a discretization of $\int_{\Gamma_{c}}\left\|g_{t}(t, \mathbf{x})\right\|_{2}^{2} \mathrm{~d} \mathbf{x}$. The matrix $\mathbf{L}_{2}$ is such that $g(t)^{\mathrm{T}} \mathbf{L}_{2} g(t)$ is a discretization of $\int_{\Gamma_{c}}\|g(t, \mathbf{x})\|_{2}^{2}+\|\nabla g(t, \mathbf{x})\|_{2}^{2} \mathrm{~d} \mathbf{x}$ or of $\int_{\Gamma_{c}}\|g(t, \mathbf{x})\|_{2}^{2}+\|\nabla g(t, \mathbf{x})\|_{2}^{2}+\|\Delta g(t, \mathbf{x})\|_{2}^{2} \mathrm{~d} \mathbf{x}$, depending on whether (13) or (14) is used. To simplify the presentation, we have assumed that $\alpha_{2}=\alpha_{3}$ and that $\alpha_{2}=\alpha_{3}=\alpha_{4}$ if $\alpha_{4}>0$. In our implementation we allow all parameters $\alpha_{1}, \ldots, \alpha_{4}$ to be different. In this section, however, distinguishing between $\alpha_{2}, \alpha_{3}$, and $\alpha_{4}$ would generate significant notational burden without providing any additional insight. The matrices $\mathbf{L}_{1}, \mathbf{L}_{2}$ also incorporate the boundary conditions $g(t, x)=0, x \in \partial \Gamma_{c}$. If the control space (12) and regularization term (14) are used, $\mathbf{L}_{1}, \mathbf{L}_{2}$ also incorporate the boundary conditions $\nabla g(t, x)=0$, $x \in \partial \Gamma_{c}$. Thus, $\mathbf{L}_{1}$ is a mass matrix on the boundary and $\mathbf{L}_{2}$ is the sum of a mass matrix and a stiffness matrix on the boundary. For our discretization, both matrices are symmetric positive definite.

To summarize, after a discretization in space, the optimal control problem can be written as

$$
\min J(g)=l^{f}\left(\mathbf{u}\left(t_{f}\right)\right)+\int_{t_{0}}^{t_{f}} l(\mathbf{u}(t), g(t)) \mathrm{d} t+\int_{t_{0}}^{t_{f}} \frac{\alpha_{1}}{2} g_{t}(t)^{\mathrm{T}} \mathbf{L}_{1} g_{t}(t)+\frac{\alpha_{2}}{2} g(t)^{\mathrm{T}} \mathbf{L}_{2} g(t) \mathrm{d} t
$$

subject to the compatibility condition

$$
g\left(t_{0}\right)=\mathbf{0}
$$

on the control (cf. (11), (12)), where $\mathbf{u}$ is the solution of (15), (16).

The semi-discrete version of the control spaces (10)-(12) are

$$
\mathscr{G}_{\text {ad }}=\left\{g: \int_{t_{0}}^{t_{f}} g(t)^{\mathrm{T}} \mathbf{L}_{2} g(t) \mathrm{d} t<\infty\right\}
$$

and

$$
\mathscr{G}_{\text {ad }}=\left\{g: \int_{t_{0}}^{t_{f}} g_{t}(t)^{\mathrm{T}} \mathbf{L}_{1} g_{t}(t)+g(t)^{\mathrm{T}} \mathbf{L}_{2} g(t) \mathrm{d} t<\infty\right\}
$$

where (20) corresponds to (10) and (21) corresponds to both (11) and (12) (since we do not distinguish between $\alpha_{2}, \alpha_{3}$, and $\alpha_{4}$ ). The semi-discrete control spaces (20) and (21) are equipped with inner products

$$
\left\langle g_{1}, g_{2}\right\rangle_{\mathscr{G}}=\int_{t_{0}}^{t_{f}} g_{1}(t)^{\mathrm{T}} \mathbf{L}_{2} g_{2}(t) \mathrm{d} t
$$

and

$$
\left\langle g_{1}, g_{2}\right\rangle_{\mathscr{G}}=\int_{t_{0}}^{t_{f}}\left(g_{1}\right)_{t}(t)^{\mathrm{T}} \mathbf{L}_{1}\left(g_{2}\right)_{t}(t)+g_{1}(t)^{\mathrm{T}} \mathbf{L}_{2} g_{2}(t) \mathrm{d} t
$$

respectively. In this paper we concentrate on the case $\alpha_{1}>0$. The case $\alpha_{1}=0$ can be handled similarly. For some details on the case $\alpha_{1}=0$ see Reference [22].

In the following, we will use subscripts to denote partial derivatives. For example, $\mathbf{N}_{g}(\mathbf{u}(t)$, $g(t), t)$ denotes the partial derivative of $\mathbf{N}$ with respect to $g$ and $\nabla_{g} l(\mathbf{u}(t), g(t))$ denotes the partial gradient of $l$ with respect to $g$. 


\subsection{Gradient computation for the semi-discrete problem}

For the numerical solution of (15)-(17) we need the gradient of the objective function $J$. Given an inner product $\langle\cdot, \cdot\rangle_{\mathscr{G}}$ on the space $\mathscr{G}$ of controls, the gradient (with respect to this inner product) is a function denoted by $(\nabla J(g))(t)$ such that the Fréchet-derivative $J_{g}$ of $J$ can be represented as

$$
J_{g}(g) g^{\prime}=\left\langle\nabla J(g), g^{\prime}\right\rangle_{\mathscr{G}}
$$

for all admissible variations $g^{\prime}$ of the control. The derivative $J_{g}(g) g^{\prime}$ can be computed using the Lagrangian

$$
\begin{aligned}
L\left(\mathbf{u}, g, \lambda, \lambda_{0}\right)= & l^{f}\left(\mathbf{u}\left(t_{f}\right), t_{f}\right)+\int_{t_{0}}^{t_{f}} l(\mathbf{u}(t), g(t)) \mathrm{d} t \\
& +\int_{t_{0}}^{t_{f}} \frac{\alpha_{1}}{2} g_{t}(t)^{\mathrm{T}} \mathbf{L}_{1} g_{t}(t)+\frac{\alpha_{2}}{2} g(t)^{\mathrm{T}} \mathbf{L}_{2} g(t) \mathrm{d} t \\
& +\int_{t_{0}}^{t_{f}} \lambda(t)^{\mathrm{T}}\left(\mathbf{q}(\mathbf{u}(t))_{t}+\mathbf{N}(\mathbf{u}(t), g(t), t)\right) \mathrm{d} t+\lambda_{0}^{\mathrm{T}}\left(\mathbf{u}\left(t_{0}\right)-\mathbf{u}_{0}\right)
\end{aligned}
$$

for (15)-(17).

The adjoint equations are obtained by setting the derivative of Lagrangian (25) with respect to $\mathbf{u}$ equal to zero. After integration by parts, this yields that $\lambda$ is the solution of the adjoint equation

$$
-\mathbf{M}(\mathbf{u}(t))^{\mathrm{T}} \lambda_{t}(t)=-\nabla_{\mathbf{u}} l(\mathbf{u}(t), g(t))-\mathbf{N}_{\mathbf{u}}(\mathbf{u}(t), g(t), t)^{\mathrm{T}} \lambda(t)
$$

$t \in\left[t_{0}, t_{f}\right]$, where $\mathbf{M}(\mathbf{u}(t))=(\mathrm{d} / \mathrm{d} \mathbf{u}) \mathbf{q}(\mathbf{u}(t))$, with final conditions

$$
\mathbf{M}(\mathbf{u}(t))^{\mathrm{T}} \lambda\left(t_{f}\right)=-\nabla_{\mathbf{u}} l^{f}\left(\mathbf{u}\left(t_{f}\right), t_{f}\right)
$$

Furthermore, $\lambda_{0}=\mathbf{M}\left(\mathbf{u}\left(t_{0}\right)\right)^{\mathrm{T}} \lambda\left(t_{0}\right)$.

The derivative of (17) is obtained by differentiating Lagrangian (25) with respect to $g$. Thus,

$$
\begin{aligned}
J_{g}(g) g^{\prime}= & \int_{t_{0}}^{t_{f}} \nabla_{g} l(\mathbf{u}(t), g(t))^{\mathrm{T}} g^{\prime}(t)+\alpha_{1} g_{t}(t)^{\mathrm{T}} \mathbf{L}_{1} g_{t}^{\prime}(t)+\alpha_{2} g(t)^{\mathrm{T}} \mathbf{L}_{2} g^{\prime}(t) \\
& +\lambda(t)^{\mathrm{T}} \mathbf{N}_{g}(\mathbf{u}(t), g(t), t) g^{\prime}(t) \mathrm{d} t
\end{aligned}
$$

where $\lambda$ is the solution of the adjoint equation (26), (27). To compute the gradient of (17) with respect to the inner product (23) we need to determine a function $\mathbf{k}$ such that

$$
J_{g}(g) g^{\prime}=\left\langle\mathbf{k}, g^{\prime}\right\rangle_{\mathscr{G}}=\int_{t_{0}}^{t_{f}} \mathbf{k}_{t}(t)^{\mathrm{T}} \mathbf{L}_{1}\left(g^{\prime}\right)_{t}(t)+\mathbf{k}(t)^{\mathrm{T}} \mathbf{L}_{2} g^{\prime}(t) \mathrm{d} t
$$


for all $g^{\prime}$. In this case, $\nabla J(g)=\mathbf{k}$. It is easy to check that the solution $\mathbf{k}$ of

$$
\begin{aligned}
-\mathbf{L}_{1} \mathbf{k}_{t t}(t)+\mathbf{L}_{2} \mathbf{k}(t)= & -\alpha_{1} \mathbf{L}_{1} g_{t t}(t)+\alpha_{2} \mathbf{L}_{2} g(t)+\nabla_{g} l(\mathbf{u}(t), g(t)) \\
& +\mathbf{N}_{g}(\mathbf{u}(t), g(t), t)^{\mathrm{T}} \lambda(t), \quad t \in\left[t_{0}, t_{f}\right] \\
\mathbf{k}\left(t_{0}\right)= & \mathbf{0} \\
\mathbf{k}_{t}\left(t_{f}\right)= & \alpha_{1} g_{t}\left(t_{f}\right)
\end{aligned}
$$

satisfies (29).

\subsection{Problem reformulation}

System (30) is a semi-discretization of an elliptic equation in $\Gamma_{c} \times(0, T)$. For simple time and spatial discretizations this equation can be solved using standard iterative techniques or sparse direct solvers (see, e.g., Reference [4]). However, if higher-order spatial and time discretization are applied, then the solution of (30) is cumbersome. We would like to avoid it. Moreover, we need to ensure that the discretization of the objective function (17) is consistent with the time-discretization of (15). Therefore, we reformulate (15)-(17) into an equivalent problem.

We introduce the additional variables

$$
h(t)=g_{t}(t)
$$

and

$$
w(t)=\int_{t_{0}}^{t}\left(l(\mathbf{u}(\tau), g(\tau))+\frac{\alpha_{1}}{2} h(\tau)^{\mathrm{T}} \mathbf{L}_{1} h(\tau)+\frac{\alpha_{2}}{2} g(\tau)^{\mathrm{T}} \mathbf{L}_{2} g(\tau)\right) \mathrm{d} \tau
$$

Since the time derivative is applied to the conserved variables $\mathbf{q}$ we view the primitive variables $\mathbf{u}$ as functions of the conserved variables $\mathbf{q}$. Thus, we rewrite (15)-(17) as

$$
\min \tilde{J}(h)=l^{f}\left(\mathbf{u}\left(\mathbf{q}\left(t_{f}\right)\right), t_{f}\right)+w\left(t_{f}\right)
$$

with state equations

$$
\begin{aligned}
& \mathbf{z}_{t}(t)=\mathbf{r}(\mathbf{z}(t), h(t), t), \quad t \in\left[t_{0}, t_{f}\right] \\
& \mathbf{z}\left(t_{0}\right)=\mathbf{z}_{0}
\end{aligned}
$$

where

$$
\mathbf{r}(\mathbf{z}(t), h(t), t)=\left(\begin{array}{c}
l(\mathbf{u}(\mathbf{q}(t)), g(t))+\frac{\alpha_{1}}{2} h(t)^{\mathrm{T}} \mathbf{L}_{1} h(t)+\frac{\alpha_{2}}{2} g(t)^{\mathrm{T}} \mathbf{L}_{2} g(t) \\
-\mathbf{N}(\mathbf{u}(\mathbf{q}(t)), g(t), t) \\
h(t)
\end{array}\right)
$$

and

$$
\mathbf{z}(t)=\left(\begin{array}{c}
w(t) \\
\mathbf{q}(t) \\
g(t)
\end{array}\right), \quad \mathbf{z}_{0}=\left(\begin{array}{c}
0 \\
\mathbf{q}\left(\mathbf{u}_{0}\right) \\
0
\end{array}\right)
$$


In reformulation (31)-(34) of (15)-(17) $\mathbf{z}$ plays the role of the state and $h$ plays the role of the control. We define

$$
f\left(\mathbf{z}\left(t_{f}\right), t_{f}\right)=l^{f}\left(\mathbf{u}\left(\mathbf{q}\left(t_{f}\right)\right), t_{f}\right)+w\left(t_{f}\right)
$$

so that objective (31) can be written as

$$
\min \tilde{J}(h)=f\left(\mathbf{z}\left(t_{f}\right), t_{f}\right)
$$

The inner product for the new controls is given by

$$
\left\langle h_{1}, h_{2}\right\rangle=\int_{t_{0}}^{t_{f}} h_{1}(t)^{\mathrm{T}} \mathbf{L}_{1} h_{2}(t) \mathrm{d} t
$$

The Lagrangian function for (32) and (36) is given by

$$
L\left(\mathbf{z}, h, \pi, \pi_{0}\right)=f\left(\mathbf{z}\left(t_{f}\right), t_{f}\right)+\pi_{0}^{\mathrm{T}}\left(\mathbf{z}\left(t_{0}\right)-\mathbf{z}_{0}\right)+\int_{t_{0}}^{t_{f}} \pi(t)^{\mathrm{T}}\left(\mathbf{z}_{t}(t)-\mathbf{r}(\mathbf{z}(t), h(t), t)\right) \mathrm{d} t
$$

The adjoint equations are

$$
\begin{aligned}
& \left.-\pi_{t}(t)=\mathbf{r}_{\mathbf{z}}(\mathbf{z}(t), h(t), t)\right)^{\mathrm{T}} \pi(t), \quad t \in\left[t_{0}, t_{f}\right] \\
& \pi\left(t_{f}\right)=-\nabla_{\mathbf{z}} f\left(\mathbf{z}\left(t_{f}\right), t_{f}\right)
\end{aligned}
$$

Furthermore, $\pi_{0}=\pi\left(t_{0}\right)$.

The gradient $\nabla \tilde{J}(h)$ satisfies

$$
\left\langle\nabla \tilde{J}(h), h^{\prime}\right\rangle=L_{h}\left(\mathbf{z}, h, \pi, \pi_{0}\right) h^{\prime} \quad \forall h^{\prime}
$$

where

$$
L_{h}\left(\mathbf{z}, h, \pi, \pi_{0}\right) h^{\prime}=\int_{t_{0}}^{t_{f}}-\pi(t)^{\mathrm{T}} \mathbf{r}_{h}(\mathbf{z}(t), h(t), t) h^{\prime}(t) \mathrm{d} t
$$

(cf. (38)). Since the inner product is given by (37), the gradient $(\nabla \tilde{J}(h))$ is determined from

$$
\int_{t_{0}}^{t_{f}}(\nabla \tilde{J}(h))(t)^{\mathrm{T}} \mathbf{L}_{1} h^{\prime}(t) \mathrm{d} t=\int_{t_{0}}^{t_{f}}-\pi(t)^{\mathrm{T}} \mathbf{r}_{h}(\mathbf{z}(t), h(t), t) h^{\prime}(t) \mathrm{d} t \quad \forall h^{\prime}
$$

i.e.

$$
(\nabla \tilde{J}(h))(t)=-\mathbf{L}_{1}^{-1} \mathbf{r}_{h}(\mathbf{z}(t), h(t), t)^{\mathrm{T}} \pi(t)
$$

If we set $\pi=(\theta, \lambda, \psi)^{\mathrm{T}}, \mathbf{u}(t)=\mathbf{u}(\mathbf{q}(t))$, and use (33) and (35), then the adjoint equation (39) is

$$
\begin{aligned}
-\theta_{t}(t) & =0 \\
-\mathbf{M}(\mathbf{u}(t))^{\mathrm{T}} \lambda_{t}(t) & =\nabla_{\mathbf{u}} l(\mathbf{u}(t), g(t)) \theta(t)-\mathbf{N}_{\mathbf{u}}(\mathbf{u}(t), g(t), t)^{\mathrm{T}} \lambda(t) \\
-\psi_{t}(t) & =\alpha_{2} \mathbf{L}_{2} g(t) \theta(t)+\nabla_{g} l(\mathbf{u}(t), g(t)) \theta(t)-\mathbf{N}_{g}(\mathbf{u}(t), g(t), t)^{\mathrm{T}} \lambda(t)
\end{aligned}
$$


for $t \in\left[t_{0}, t_{f}\right]$ with final conditions

$$
\left(\begin{array}{c}
\theta\left(t_{f}\right) \\
\lambda\left(t_{f}\right) \\
\psi\left(t_{f}\right)
\end{array}\right)=\left(\begin{array}{c}
-1 \\
-\mathbf{M}(\mathbf{u}(t))^{-T} \nabla_{\mathbf{u}} l^{f}\left(\mathbf{u}\left(t_{f}\right), t_{f}\right) \\
\mathbf{0}
\end{array}\right)
$$

Using the definition (33) of $\mathbf{r}$ and $\pi=(\theta, \lambda, \psi)^{\mathrm{T}}$, we see that gradient (41) is given by

$$
(\nabla \tilde{J}(h))(t)=-\mathbf{L}_{1}^{-1}\left(\alpha_{1} \theta(t) \mathbf{L}_{1} h(t)+\psi(t)\right)
$$

We conclude this section with a comparison of the original formulation (15)-(17) and reformulation (31), (32). In reformulation (31), (32) the optimization variable is $h=g_{t}$. After a time discretization is performed, this corresponds to a scaling of the optimization variable such as those described in Reference [35, Section 7.1]. If $g_{*}$ is the solution of (15)-(17), then $h_{*}=\left(g_{*}\right)_{t}$ solves (31), (32). In this sense the reformulation does not change the solution of the problem. However, if a gradient method or a non-linear conjugate gradient method is used, then the iterates are not scaling invariant and such optimization algorithms will approach the solution along a different path depending on the formulation used. In the context of the gradient method (the first step of a non-linear conjugate gradient method is a gradient step) this means that if $g_{+}=g-\gamma \nabla J(g)$ is the new iterate generated by the gradient method applied to (15)-(17) and if $h_{+}=h-\gamma \nabla \tilde{J}(h)$ is the new iterate generated by the gradient method applied to (31), (32), then $h_{+} \neq\left(g_{+}\right)_{t}$ even if $h=(g)_{t}$ (see Reference [35, Section 7.1]). The same is also true if a quasi-Newton method is used to solve the optimization problems, unless the quasi-Newton matrices are properly scaled (see References [4, Section 8.3]; [36, Appendix]).

\section{TIME-DISCRETIZATION}

For the discretization of the state equation, we use the classical fourth-order explicit RungeKutta method. The formulation of the fully discretized problem from is rather standard and follows procedures that are well known from the optimal control of ordinary differential equations. However, there are a few subtle issues that concern the computation of the gradients for the fully discrete problem and the computation of the controls when higher-order RungeKutta time-stepping schemes are used. We refer to [37] for a general treatment and to [22] were a discussion of these issues for the semi-discrete problem (18) or (32), (36) can be found.

\section{OPTIMIZATION ALGORITHM}

Our numerical results are produced using a non-linear conjugate gradient algorithm for the solution of the fully discretized problem. Our implementation follows $[38,39]$ with the exception that we use the inner products that are discretizations of (22) or (23), respectively. The inner product acts as a preconditioner within the non-linear conjugate gradient algorithm. Thus, it effects the convergence behaviour of the algorithms, but it does not alter the solution of the problem. Choosing discretizations of (22) or (23) instead of the standard Euclidean 
inner product minimizes the mesh-dependent behaviour of the algorithms and reduces artificial ill-conditioning due to discretization.

As in Reference [11] we observed that the objective function is rapidly reduced in the first 15-25 iterations (not counting inner iterations needed to determine the step size) with diminishing returns thereafter. In Section 7.1 we document in more detail the optimization iteration for a representative objective function. In addition to the non-linear conjugate gradient algorithm, we have also experimented with the limited memory BFGS methods (e.g., Reference [39]), and even extended the latter algorithm to handle bound constraints on the control. Those results will be presented elsewhere. See Reference [40] for related results.

To cope with the very large size of state and control variables, we write the state to disk after a fixed number of time steps has been performed. More sophisticated storage management techniques, which will be considered in the future, are discussed in References [1,7,41].

We remark that the solution of one adjoint equation, where spatial adjoints are computed using the $\mathrm{AD}$ tool TAMC [42] is about four times as expensive as the solution of one state equation (compressible Navier-Stokes equation). One reason for the higher expense of the adjoint solve is that state information has to be read from disk before a time-slice of the adjoint can be computed. All computations are performed in parallel using OpenMP on the SGI Origin 2000. OpenMP directives are added by hand to the state routines and extensive tuning and testing has been performed to optimize both single and multiple processor performance. Since OpenMP is not currently supported by TAMC, the appropriate OpenMP directives are added by hand to the TAMC produced adjoint code. The runs presented here are typically performed on 8 processors with a measured speed-up of about 5.2 for both the state and adjoint computations.

\section{RESULTS}

The optimal control formulation described above is now applied to the vortex-wall interaction problem. The initial flow field is determined by the superposition of two compressible Oseen vortices [43]. The initial vortex core radii are $L^{*}$ and the maximum azimuthal velocity $v_{\theta_{m}}^{*}$ at the edge of the viscous core of each vortex at the initial time are the length and velocity scales chosen for the non-dimensionalization of the Navier-Stokes equations. Thus, the reference Reynolds and Mach numbers are defined as

$$
R e=\frac{\rho_{\infty}^{*} L^{*} v_{\theta_{m}}^{*}}{\mu_{\infty}^{*}}, \quad M=\frac{v_{\theta_{m}}^{*}}{\sqrt{\gamma R^{*} T_{\infty}^{*}}}
$$

where $\rho_{\infty}^{*}, \mu_{\infty}^{*}$, and $T_{\infty}^{*}$ are the density, viscosity, and temperature far from the vortices; $R^{*}$ is the gas constant; and ' $*$ ' denotes dimensional quantities.

Our computational domain is $[-15,15] \times[0,15]$ in non-dimensional units and at time $t=0$ the vortices are centred at $( \pm 2,7.5)$. Although each individual Oseen vortex is a solution to the Navier-Stokes equations, the superposed field does not satisfy the equations of motion due to the non-linearity of the convective term and it also fails to satisfy the wall no-slip and thermal boundary conditions. Hence, the flow is advanced a number of time steps until time $t_{0}$ to let the transients associated with these incompatibilities leave the domain through the top and sides where a non-reflecting boundary treatment is used. The resulting field at time $t_{0}$ is taken as the initial condition to our problem. The Mach, Reynolds, and Prandtl numbers 


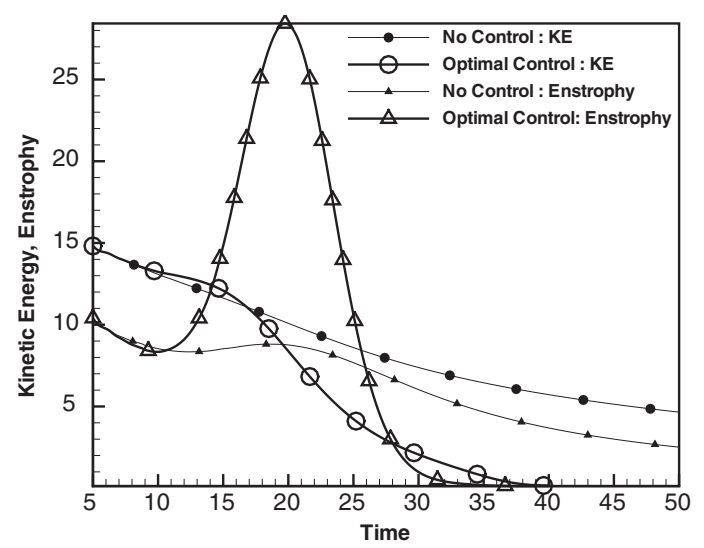

Figure 1. The evolution of integrated kinetic energy and enstrophy with time for uncontrolled and TKE control, run (a).

are $M=0.5, R e=25, \operatorname{Pr}=1$, respectively. As stated in the introduction, for the demonstration problems presented here, constant Prandtl number and fluid properties (viscosities and thermal conductivity) are assumed along with Stokes hypothesis for the second coefficient of viscosity, $\lambda=-2 \mu / 3$.

In all cases, the control $g$ is the wall-normal velocity which is a function of the wall co-ordinate and time. Positive $g$ represents injection (blowing) of fluid into the domain while negative $g$ corresponds to suction of fluid out of the domain (cf. Equation (5)). It is assumed that the injected/ingested fluid is always in thermal equilibrium with the solid surface.

Before presenting the control results, we first document the uncontrolled dynamics by considering the interaction of the vortex pair with a no-slip, adiabatic wall with inviscid characteristic based far-field boundary treatments used on the top and sides. In the uncontrolled flow, the vortex pair convects downward due to the self-induced velocity eventually interacting with the wall at the lower boundary. Since the Reynolds number is rather low, the vortices decay as shown in Figure 1 by the evolution of integrated kinetic energy $\int_{\Omega} \rho \mathbf{v}^{\mathrm{T}} \mathbf{v} / 2 \mathrm{~d} \Omega$ and integrated enstrophy $\int_{\Omega} \boldsymbol{\omega}^{\mathrm{T}} \boldsymbol{\omega} / 2 \mathrm{~d} \Omega$, where $\boldsymbol{\omega}=\nabla \times \mathbf{v}$ is the vorticity vector. The integrated kinetic energy undergoes a monotonic decay as dissipation converts kinetic energy into internal energy. While the integrated enstrophy also generally decays, when the vortices approach the wall, secondary vorticity is generated through the no-slip condition leading to a local maximum in integrated enstrophy at approximately $t=22$. In all controlled runs, the integrated enstrophy peak of the uncontrolled flow lies well within the optimization time window.

For all computations, we use a mesh with 128 grid points in the $x$-and $y$-directions. A uniform mesh is used in the $x$-direction while the mesh is clustered in the $y$-direction near the wall using an algebraic stretching function in order to better capture wall-normal gradients. The resulting mesh size $\Delta y$ at the wall is 0.37 times that of a uniform mesh, i.e. $\Delta y=0.37 \times 15 / 128$. The time step is $\Delta t=0.025$. To compute the initial conditions, the flow equations are advanced for 600 time steps to minimize effects resulting from the startup transient. Unless otherwise stated, optimal controls are computed over 800 time steps, i.e. $t_{0}=15$ to $t_{f}=35$ such that the enstrophy peak of the uncontrolled flow lies within this time window, see Figure 1. 
Table I. Regularization parameters and objective function values used in the boundary control of terminal kinetic energy.

\begin{tabular}{lccccccc}
\hline Run & $\alpha_{1}$ & $\alpha_{2}$ & $\alpha_{3}$ & $\alpha_{4}$ & $J_{0}$ & $J_{\text {final }}$ & $T K E_{\text {final }}$ \\
\hline $\mathrm{a}$ & 0 & 0.005 & 0.005 & 0 & 12.43 & 0.24 & 0.20 \\
$\mathrm{~b}$ & 0.05 & 0.005 & 0.005 & 0 & 12.43 & 0.25 & 0.20 \\
$\mathrm{c}$ & 0.05 & 0.005 & 0.005 & 0 & 12.43 & 0.48 & 0.42 \\
$\mathrm{~d}$ & 0.05 & 0.005 & 0.005 & 0.05 & 12.43 & 0.28 & 0.22 \\
\hline
\end{tabular}

\subsection{Optimal control of terminal kinetic energy}

The objective functional for this test case is given by (6) with (7) and $\Omega_{0}=[-15,15] \times[0,15]$ (the entire computational domain). We consider two control boundaries. In the first case, the control boundary is the entire lower wall

$$
\Gamma_{c}=\left\{\mathbf{x}=\left(x_{1}, 0\right): x_{1} \in[-15,15]\right\}
$$

In the second case, we restricted the control boundary to be

$$
\Gamma_{c}=\left\{\mathbf{x}=\left(x_{1}, 0\right): x_{1} \in[-11.25,11.25]\right\}
$$

We performed four runs. Runs (a) and (b) use the control boundary (45) while runs (c) and (d) use (46). The coefficients $\alpha_{j}$ are documented for all runs in Table I. In Table I, $J_{0}$ refers to the objective function at the initial control $g=0, J_{\text {final }}$ denotes the value of the objective function at the computed optimal control and $T K E_{\text {final }}$ refers to the value of (7) at the computed optimal control. In Runs (a) and (c) the non-linear conjugate gradient method was truncated early after 15 iterations, because temporal or spatial oscillations arose and were increased in following non-linear conjugate gradient iterations.

We begin by examining runs (a) and (b), since detailed results for these cases have been reported in Reference [22]. Here, we focus only on the computed controls as shown in Figure 2. These plots clearly demonstrate the effect of the regularization term $\int \alpha_{1} / 2\left\|g_{t}\right\|_{2}^{2}$. In run (a), without time regularization, the control starts to oscillate in time in the second half of $\left[t_{0}, t_{f}\right]$ and also exhibits a large jump at $t_{0}$. We also note that setting $\alpha_{1}=\alpha_{3}=0$ (not shown here) also produces strong spatial and temporal oscillations in the control which frequently led to a failure in the compressible Navier-Stokes solver. Such jumps and oscillations are also physically undesirable since they would place severe demands on actuator performance.

With time regularization, in run (b), the control is primarily localized in the centre of the domain near the region of vortex/wall interaction and consists primarily of suction although there are small amounts of blowing visible at late times near $x= \pm 7$. The main effect of the control is to absorb the vortices through the wall as discussed in Reference [22] and evident in the evolution of kinetic energy and enstrophy in Figure 1.

For both runs (a) and (b) no second-order spatial regularization of the control is used. In Reference [22] it was suggested that the control space (11) and corresponding regularization term (13) may be sufficient for this control boundary. However, scrutiny of Figure 2(b), shows a tendency for sharp gradients to appear in the control near $x= \pm 15$ where there is an incompatibility between boundary conditions on the sides and bottom. Since we use one-sided finite differences near the boundaries (see Section 3) the impact on the interior of the solution 


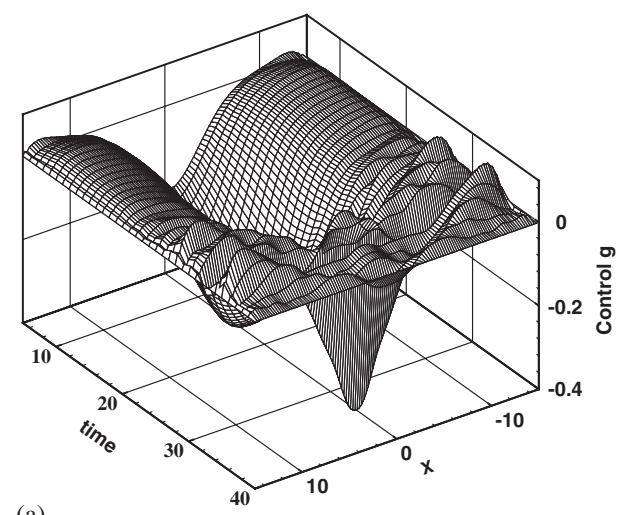

(a)
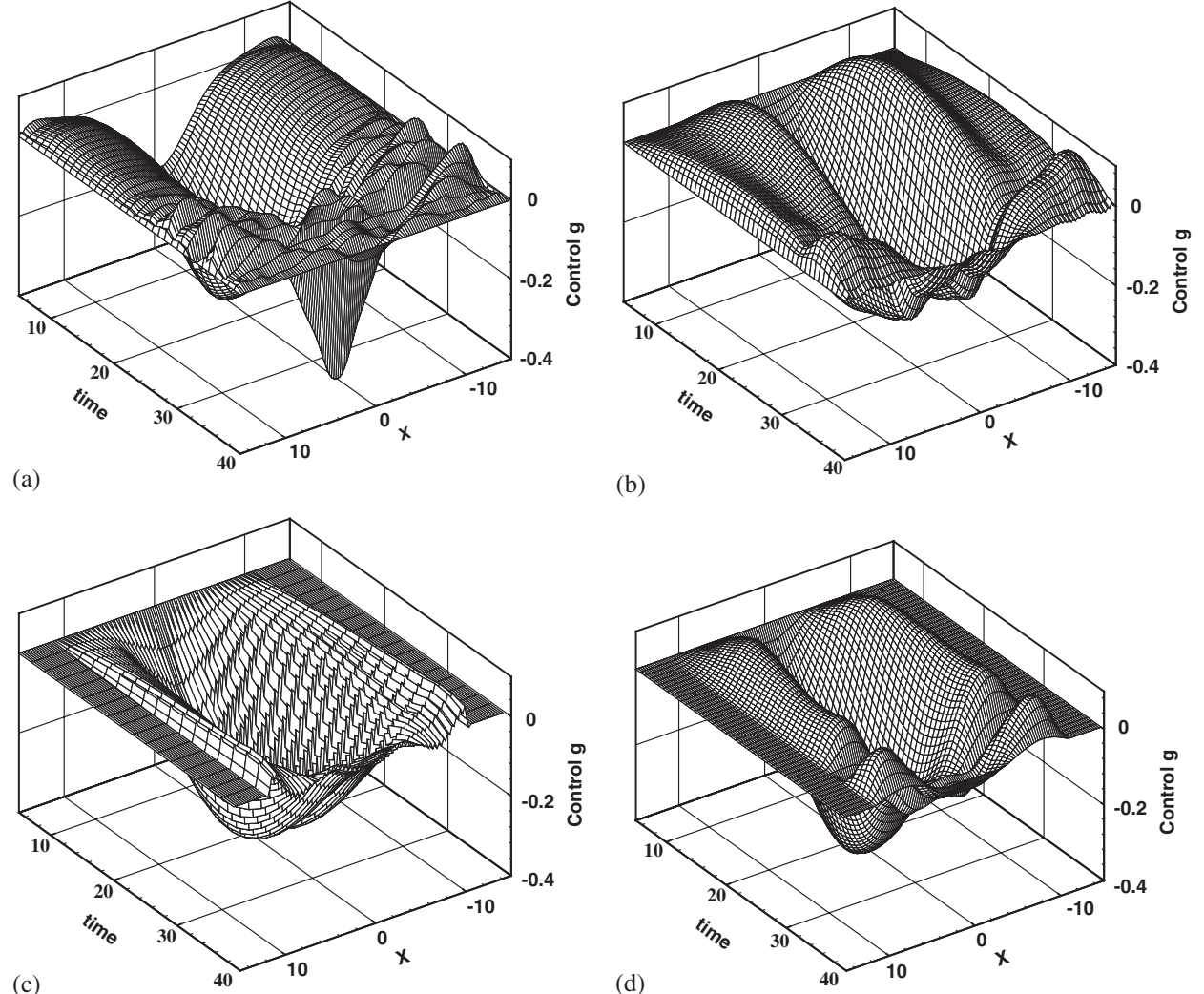

(b)

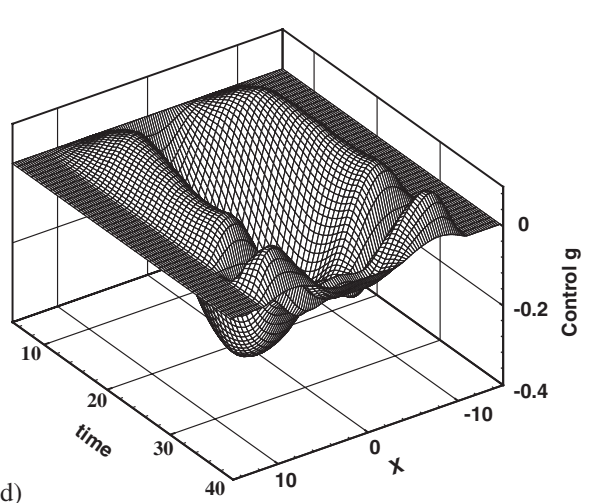

Figure 2. Optimal wall-normal velocity distributions for TKE control: (a) $\alpha_{1}=0$, $\alpha_{2}=\alpha_{3}=0.005, \alpha_{4}=0$; (b) $\alpha_{1}=0.05, \alpha_{2}=\alpha_{3}=0.005, \alpha_{4}=0$; (c) $\alpha_{1}=0.05, \alpha_{2}=\alpha_{3}=0.005$, $\alpha_{4}=0$; and (d) $\alpha_{1}=0.05, \alpha_{2}=\alpha_{3}=0.005, \alpha_{4}=0.05$.

is minor although very small grid-dependent oscillations are present in the interior suggesting that additional spatial regularity would be beneficial.

To explore the impact of second derivative regularization, runs (c) and (d) have been performed using control boundary (46) in which the control is limited to a region near the center of the computational domain. This choice of control boundary is motivated by the control distributions in run (b) for which the control is primarily localized near the center of the domain. This control boundary also removes the potential for mismatch between side and bottom boundary conditions which occurred in runs (a) and (b). The coefficients $\alpha_{j}$ for runs (c) and (d) are documented in Table I. With this control boundary, node-to-node oscillations develop when second derivative regularization is not used $\left(\alpha_{4}=0\right.$ shown in Figure 2(c)) since the localized boundary control leads to sudden spatial gradients on the bottom boundary that are incompatible with the fourth-order finite difference scheme used in the interior. The sharp gradients and spatial oscillations are suppressed if the second spatial derivative of the control is also penalized, i.e. if $\alpha_{4}>0$, as done in run (d) and shown in Figure 2(d). Note that by using both first and second-derivative regularization, we are able to enforce both continuity of $g$ and $g_{s}$ on $\partial \Gamma_{c}$. 


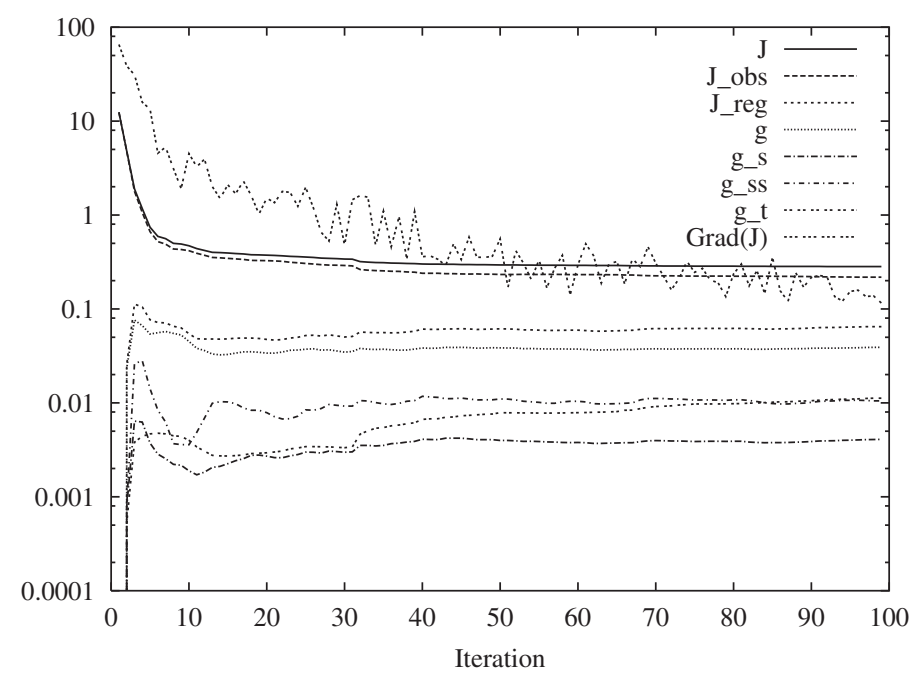

Figure 3. Optimization history for TKE case (d).

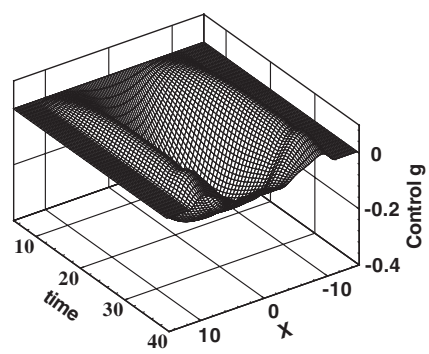

(a)

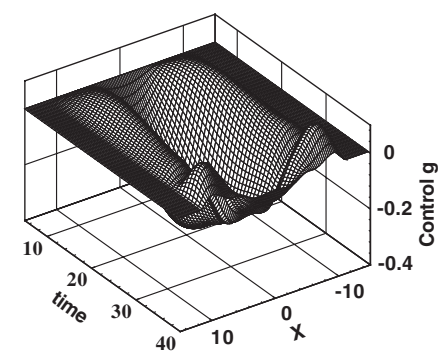

(b)

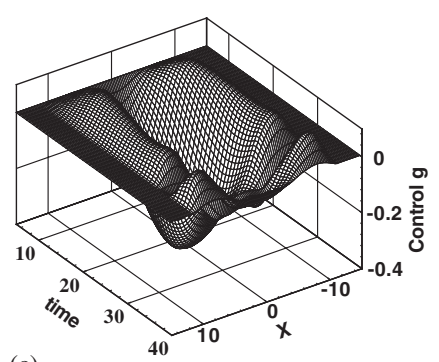

(c)

Figure 4. Optimal wall-normal velocity distributions for TKE control case (d): (a) 16 iterations; (b) 32 iterations; and (c) 60 iterations.

The use of control boundary (46) along with second derivative regularization does not lead to significant qualitative differences in either the control distributions shown in Figure 2(d) or the overall reduction in terminal kinetic energy as shown in Table I. Run (b) results in a 62 times reduction in kinetic energy while case (d) yields a 56 times reduction.

A typical optimization history is given in Figure 3 which shows the evolution of the objective function as well as each of the terms which comprise it for case (d). As indicated in Section 6, the most rapid decrease in the objective function occurs in the first 15-25 iterations of the non-linear conjugate gradient algorithm, similar to that reported by Reference [11]. After about 30 iterations, the major features of the control have been determined and Figure 4(a), (b) shows the control distribution at 16 and at 32 iterations, respectively. Additional iterations lead to more subtle changes in the control as seen in Figure 4(c) which shows the control distribution after 60 iterations. These changes in the control generally come at the expense of increases in space-time gradients as is clearly seen in the $\int\|\nabla g\|^{2}, \int\|\Delta g\|^{2}$, and 
$\int\left\|g_{t}\right\|^{2}$ traces in Figure 3. However, by 50 iterations, relative changes in the various terms in the objective function are very small and the control distribution at iteration 60 is nearly indistinguishable from that of iteration 90 in Figure 4(d). From an engineering point of view, the control at, 16 iterations may be more desirable than that at 60 or 90 , since the performance (in terms of reduction in $J_{\text {obs }}$ ) is nearly the same and the peak magnitude and space/time gradients are less. Of course, with the space/time regularization terms we have introduced, one could alter the relative values of $\alpha_{1}, \alpha_{2}, \alpha_{3}$ and $\alpha_{4}$ to achieve a desired change in the control distributions although this becomes more an issue of control design. The regularization parameters for this and the following examples were chosen by trial and error. More systematic selections of these parameters are discussed in References [11,41] and the references cited therein. Finally, we point out, that for all the optimal control simulations we have performed, the space/time gradients of the control tend to increase (albeit slightly) as more iterations are taken and this is in agreement with the observations of [11]. This has an important implication for highly oscillatory controls, such as case (a) and (c), which resulted from insufficient regularization - the severe oscillations will only get worse if additional conjugate gradient iterations are taken. For cases such as (d), which include the full regularization term, we have performed extended iterations to ensure that no grid-dependent oscillations appear.

We conclude this section by reiterating that the control space (12) and corresponding regularization term (14) are required when the control region is only a subset of the boundary and is also likely beneficial in the case where control is applied over the entire bottom boundary, although we did not explore that further here. In both cases, sharp spatial derivatives in the boundary conditions at $\partial \Gamma_{c}$ lead to oscillations in the computed results. These results indicate that the interaction of the control boundary and the spatial discretization come to bare on the choice of the control space and corresponding regularization term. For the high accuracy, low dissipation numerical methods commonly used in aeroacoustics simulations, sufficient control regularity must be enforced to ensure the consistency, smoothness, and realizability of computed controls.

\subsection{Optimal control of heat transfer}

The motivation for this test case is to investigate the possibility of controlling the heat transfer across an isothermal wall in a time interval of interest by applying unsteady wall-normal velocity control. This test problem demonstrates how the coupling of thermodynamic and kinematic flow variables through the compressible Navier-Stokes equations can be used to effectively control one with the other.

The objective functional is given by (6) with (8). The regularization term in (6) is given by (13) or by (14). Suction/blowing is applied only on the portion $\Gamma_{c}=\left\{\mathbf{x}=\left(x_{1}, 0\right): x_{1} \in\right.$ $[-11.25,11.25]\}$ of the lower wall $\Gamma=\left\{\mathbf{x}=\left(x_{1}, 0\right): x_{1} \in[-15,15]\right\}$. Moreover, the normal derivative is only controlled over the same part of the lower boundary, i.e. $\Gamma_{0}=\Gamma_{c}$ in (8). The weighting function $\omega$ is a smooth function which is zero at $x_{1}= \pm 11.25$ and one on $[-9,9]$. The term $\omega \partial T / \partial n$ in the objective contributes to adjoint boundary conditions on $\Gamma_{0} \subset \Gamma$, but not outside $\Gamma_{0}$. If the weighting were $\omega \equiv 1$, then the adjoint boundary conditions can and usually do exhibit jumps at $\mathbf{x}=( \pm 11.25,0)$. The addition of the smooth weighting function with $\omega( \pm 11.25)=0$ avoids these jumps in the adjoint boundary conditions.

The lower wall is isothermal while an inviscid characteristic based far-field boundary condition is imposed on the other boundaries. The temperature boundary condition at the lower wall 
Table II. Regularization parameters used in the boundary control of wall-normal temperature.

\begin{tabular}{lcccc}
\hline Run & $\alpha_{1}$ & $\alpha_{2}$ & $\alpha_{3}$ & $\alpha_{4}$ \\
\hline $\mathrm{a}$ & 0 & 0.5 & 0.5 & 0 \\
$\mathrm{~b}$ & 0.5 & 0.5 & 0.5 & 0 \\
$\mathrm{c}$ & 0.5 & 0.5 & 0.5 & 0.05 \\
\hline
\end{tabular}

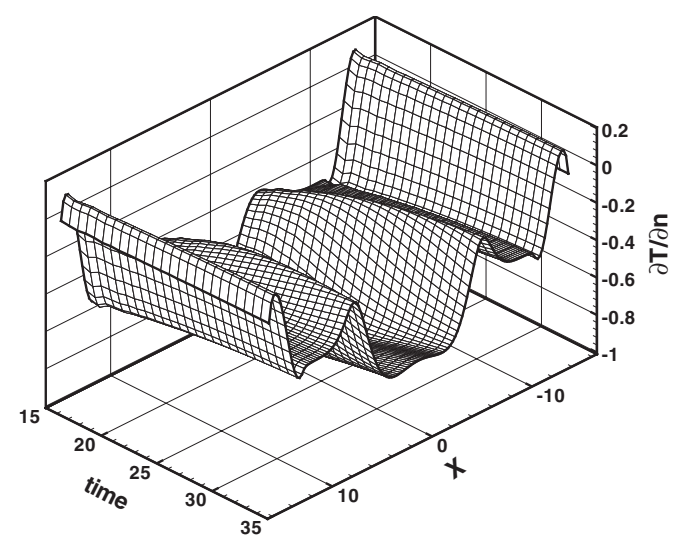

Figure 5. Distribution of wall normal temperature gradient, $\partial T / \partial n$, at the wall for the uncontrolled flow.

is $T(\mathbf{x}, t)=T_{b}(\mathbf{x}), \mathbf{x} \in \Gamma_{c}$, where $T_{b}$ is a continuously differentiable function with $T_{b}(\mathbf{x})=1.5$, $\mathbf{x} \in \Gamma_{c}$ and $T_{b}(\mathbf{x})=2, \mathbf{x}=( \pm 15,0)$. This temperature profile ensures compatibility with the far-field temperature at $( \pm 15,0)$.

In our first two runs we use the control space (11) and the regularization term (13). The last run uses the control space (12) and the regularization term (14) The first run does not include a regularization of the time derivative of the control, the other two do. The coefficients $\alpha_{j}$ in the regularization term for the runs are given in Table II.

The uncontrolled flow is given in Figure 5 which shows the wall-normal temperature gradient $\partial T / \partial n=-\partial T / \partial x_{2}$ on the bottom wall that is proportional to the heat transfer rate from the fluid to the wall, $\dot{q}=-\kappa \partial T / \partial n$. Since $\mathbf{n}$ is the outward unit normal, $\partial T / \partial n>0$ denotes heat transfer from the wall to the fluid. Since the uncontrolled wall is cool, the nominal heat transfer is from the flow to the wall as indicated in Figure 5 by the predominately negative $\partial T / \partial n$. Since the vortex cores are lower in temperature than the ambient fluid, there is a local reduction in heat transfer near the vortex core locations which can be easily tracked in Figure 5.

Heat transfer results for the controlled flows are shown in on the right side of Figure 6. Keep in mind that we only control $\partial T / \partial n$ over $\Gamma_{c}=\left\{\mathbf{x}=\left(x_{1}, 0\right): x_{1} \in[-11.25,11.25]\right\}$. In this region of the wall, $(\partial T / \partial n)^{2}$ is reduced nicely by all controls. For run (a), where no timederivative regularization term $\left(\alpha_{1}=0\right)$ is enforced, $(\partial T / \partial n)^{2}$ is large for a small time near $t_{0}$. Actually in that time region for this run, the controlled $(\partial T / \partial n)^{2}$ is larger than in the 

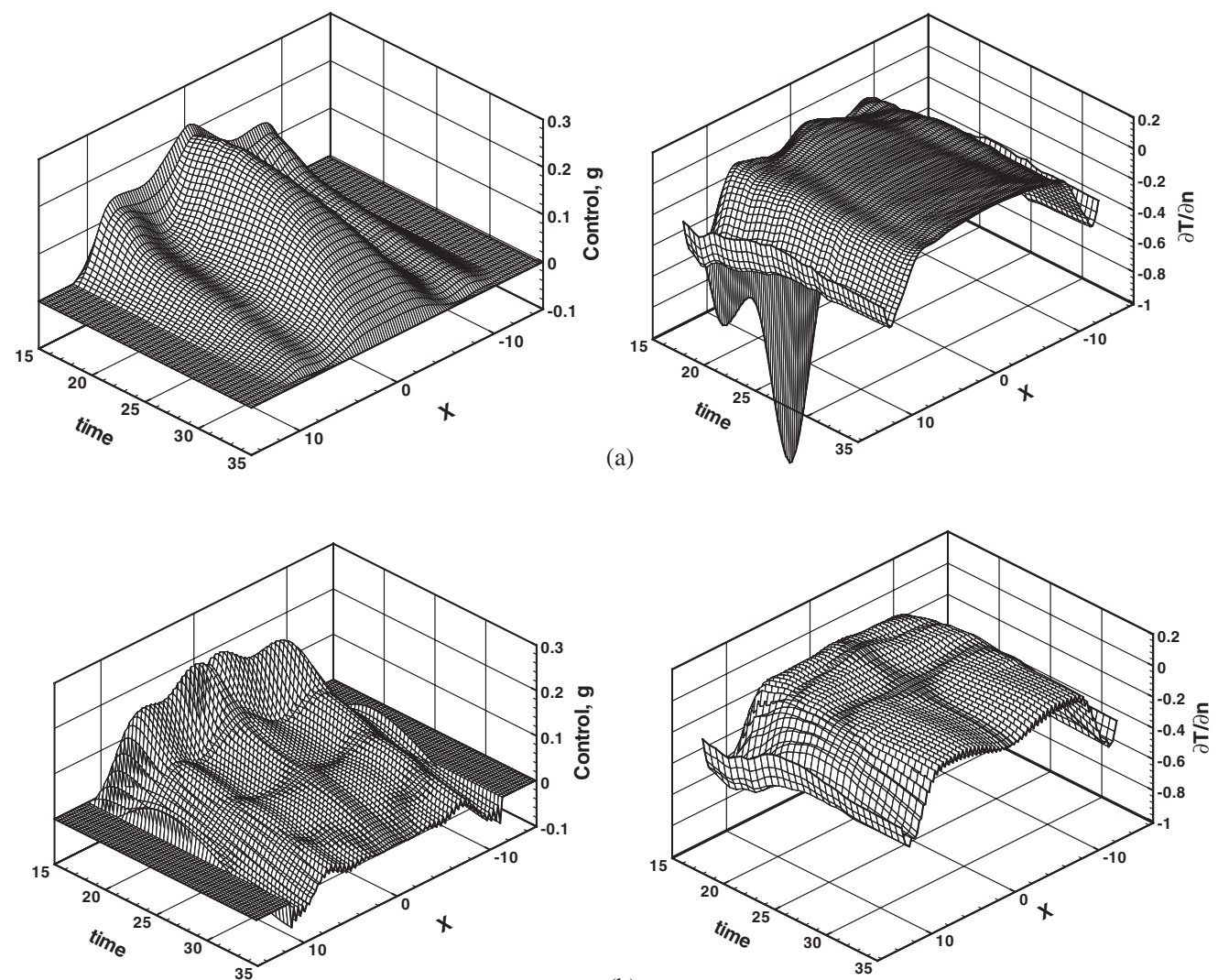

(b)
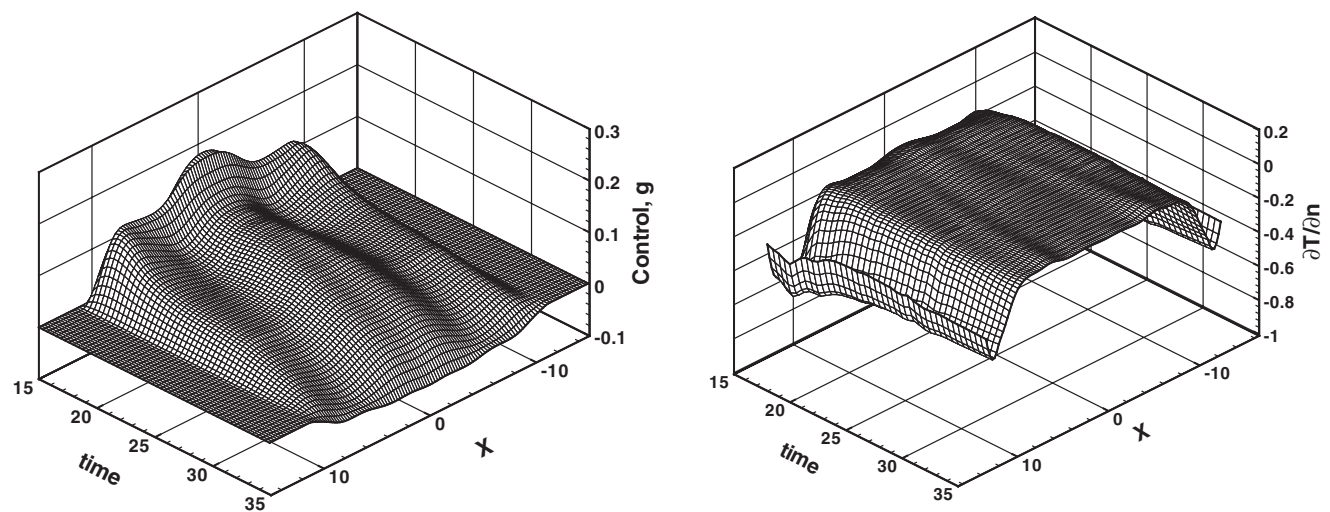

(c)

Figure 6. Distributions of control (left) and wall normal temperature gradient, $\partial T / \partial n$, at the wall (right) for flows with heat transfer control: (a) $\alpha_{1}=0, \alpha_{2}=\alpha_{3}=0.5, \alpha_{4}=0$; (b) $\alpha_{1}=\alpha_{2}=\alpha_{3}=0.5, \alpha_{4}=0$; and (c) $\alpha_{1}=\alpha_{2}=\alpha_{3}=0.5, \alpha_{4}=0.05$. 
Table III. Objective function values for different regularization parameters.

\begin{tabular}{lccccccc}
\hline Run & $J_{0}$ & $J_{\text {final }}$ & $\frac{1}{2} \int \omega(\partial T / \partial n)^{2}$ & $\alpha_{1} / 2 \int\left\|g_{t}\right\|_{2}^{2}$ & $\alpha_{2} / 2 \int\|g\|_{2}^{2}$ & $\alpha_{3} / 2 \int\|\nabla g\|_{2}^{2}$ & $\alpha_{4} / 2 \int\|\Delta g\|_{2}^{2}$ \\
\hline a & 53.970 & 3.650 & 2.560 & - & 1.034 & 0.057 & - \\
b & 53.970 & 4.100 & 3.364 & 0.097 & 0.593 & 0.046 & - \\
c & 53.970 & 3.608 & 2.792 & 0.301 & 0.486 & 0.025 & 0.004 \\
\hline
\end{tabular}

no-control flow. Nevertheless, the control substantially reduces the integral (8) in all cases (see Table III). The sudden changes in the wall-normal temperature gradient for the runs without time regularization are directly related to sudden changes in the controls for these flows.

The computed controls are shown on the left-hand side of Figure 6. Since control is only applied over $\Gamma_{c}$ the wall-normal velocity is zero elsewhere. We see that the structure of the optimal controls computed using a time derivative regularization $\left(\alpha_{1}>0\right)$ is fundamentally different from those computed without time derivative regularization $\left(\alpha_{1}=0\right)$. This follows already from the optimality conditions $\nabla J(g)=0$ using (26), (30) for the case $\alpha_{1}>0$ and analogous equations for the case $\alpha_{1}=0$. The computed controls for $\alpha_{1}=0$ change rapidly in time near $t_{0}$ and near $t_{f}$ and they exhibit slight oscillations elsewhere in time.

The controls without time regularization can exhibit large temporal derivatives that not only may cause numerical difficulties but which would also place undue demands on the design of physical actuators for problems of this type. A regularization of the time derivative of the controls enforces temporal smoothness. However, close inspection of the optimal control computed with time regularization in run (b) reveals slight spatial oscillations. See plot (b) in Figure 6. Moreover, all optimal controls may increase sharply near the endpoints $x_{1}= \pm 11.25$ of the control boundary $\Gamma_{c}$. To suppress oscillations and to avoid a sharp increase in the horizontal velocity near the boundary points $\left(x_{1}, x_{2}\right)= \pm(11.25,0)$ we switch to the control space (12) and the regularization term (14). The regularization parameters for the additional run (c) is listed in Table II. The optimal controls for this run are shown in plot (c) of Figure 6. We see that spatial oscillations are suppressed and the spatial transition from the no-control to the control boundary is smoother than in run (b). The additional control regularization has only a minor effect on the total heat transfer as can be seen in Figure 6.

Table III shows the values of the objective functional (6) with (8) at the initial iterate, i.e. zero control $\left(J_{0}\right)$ and at the final control iterate, $\left(J_{\text {final }}\right)$, in addition to the values of the (8) and those for the individual regularization terms at the final control. Overall, the square magnitude of the heat transfer is typically reduced by approximately $93 \%$ for the controlled flows. The slight additional reduction in heat transfer for the flows without time regularization is likely due to the fact that these flows have large controls near $t_{0}$, however the sudden jump in control at $t_{0}$ not only causes oscillations in the numerical solutions, but would also require unrealistically high actuator frequency response.

Figure 6 shows that in all cases fluid is primarily injected into the domain such that the optimal control takes the form of film cooling. Temperature contours for the uncontrolled flow and controlled flow, run (a), are plotted in Figure 7. Since the fluid blown into the domain is at the wall temperature $T=1.5$, this creates a small layer of fluid with almost constant temperature $T=1.5$ near $\Gamma_{c}$, see Figures $7(\mathrm{~d})$ and $7(\mathrm{f})$, thereby reducing $\partial T / \partial n$. The 

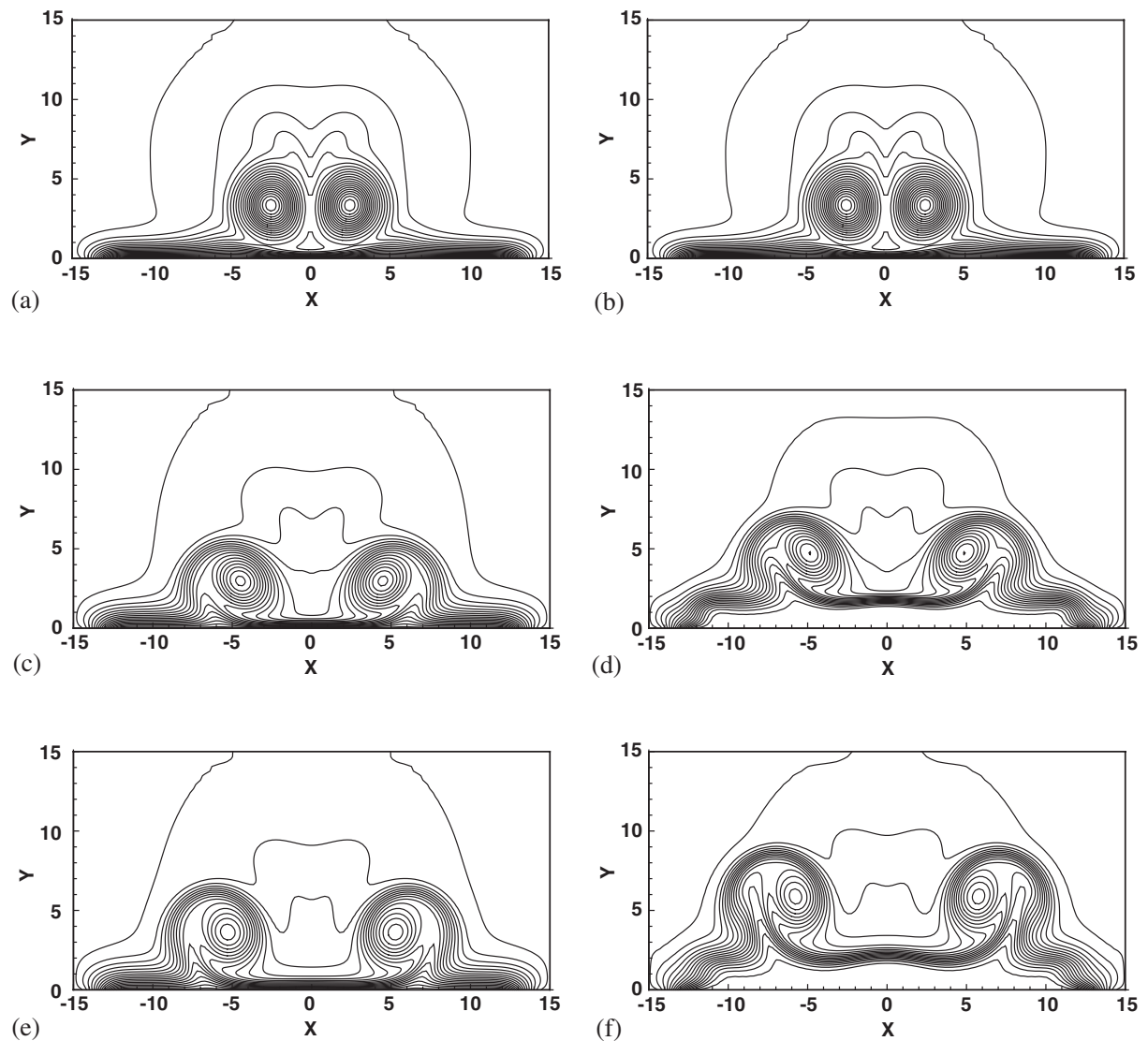

Figure 7. Temperature contours for the uncontrolled flow and heat transfer control flow, run (a). There are 25 evenly spaced contours from 1.4 to 2.1: (a) no control, $t=t_{0}$; (b) optimal control, run (a), $t=t_{0}$; (c) no control, $t=t_{0}+12.5$; (d) optimal control, run (a), $t=t_{0}+12.5$; (e) no control, $t=t_{f}$; and (f) optimal control, run (a), $t=t_{f}$.

temperature contour plots for the controlled flows in runs (b) and (c) are very similar to those shown in Figures 7(d) and 7(f) for run (a) and are not given here.

\subsection{Optimal control of wall shear stress}

In this test case, we minimize a measure of the drag force on the lower wall through wallnormal suction or blowing. Specifically, the observation part of the objective function is given by (9). Since $\mathbf{n}=(0,-1)^{\mathrm{T}}$, (9) reads

$$
J_{\text {obs }}(\mathbf{u}(g))=\frac{1}{2} \int_{t_{0}}^{t_{f}} \int_{\Gamma_{0}} \omega(\mathbf{x}) \tau_{12}^{2} \mathrm{~d} \mathbf{x}
$$

where $\tau$ is defined by (1). The computational domain, the control boundary $\Gamma_{c}$, the observation boundary $\Gamma_{0}$, and the weighting function $\omega$ in (47) are defined as in Section 7.2. The lower 
Table IV. Regularization parameters used in the boundary control of wall shear stress.

\begin{tabular}{ccccc}
\hline Run & $\alpha_{1}$ & $\alpha_{2}$ & $\alpha_{3}$ & $\alpha_{4}$ \\
\hline $\mathrm{a}$ & 0.5 & 0.5 & 0.5 & 0 \\
$\mathrm{~b}$ & 0.5 & 0.5 & 0.5 & 0.05 \\
\hline
\end{tabular}

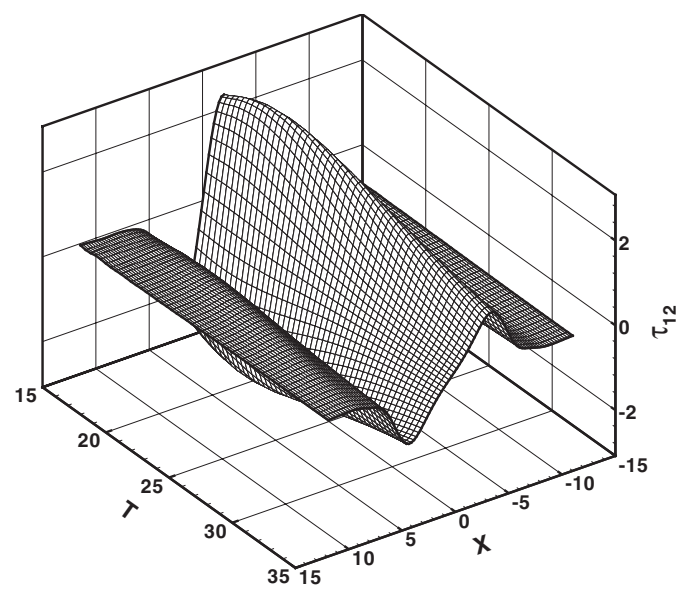

Figure 8. Distribution of wall shear stress, $\tau_{12}$, for the uncontrolled flow.

wall is adiabatic, $\partial T(\mathbf{x}, t) / \partial n=0$ on $\mathbf{x}=\left(x_{1}, 0\right)$, while an inviscid characteristic based far-field boundary condition is imposed on the other boundaries. The spatial discretization is identical to the one described in Section 7.2. As in Section 7 we advance the flow equations for 600 time steps with step size $\Delta t=0.025$ to obtain the initial conditions at $t_{0}=15$. We control from $t_{0}=15$ to $t_{f}=35$, where, in this case, 2000 time steps with step size $\Delta t=0.01$ are used.

We perform two computations with regularization of the time derivative of the control, $\alpha_{1}>0$. Without this regularization the control exhibits strong temporal oscillations. Since this behaviour is qualitatively comparable with that in Sections 7.1 and 7.2, we omit the results for $\alpha_{1}=0$. The regularization parameters are displayed in Table IV.

Temporal and spatial variations of wall shear stress on the lower wall for the uncontrolled run are depicted in Figure 8. Associated with each vortex is an extrema in local wall shear stress and at the center of the pair, $x=0$, the shear stress is zero corresponding to a stagnation point. Plots (a) and (b) of Figure 9 show the wall shear-stress on the lower wall for optimal control runs (a) and (b). Clearly, both runs are successful in reducing the magnitude of shear stress over the controlled region $\Gamma_{c}$, but (a) exhibits non-physical high-frequency oscillations in the spatial direction. These oscillations in the observation term emanate from the highly oscillatory controls of plot (a) of Figure 9. In run (b) the control is restricted to the smoother space of (12) which suppresses these oscillations very effectively and also enforces zero slope at points $x= \pm 11.25$ where the transition from the controlled boundary to the uncontrolled boundary occurs.

The qualitative effect of the optimal control on the vortex trajectories is shown by density contours for the uncontrolled flow and controlled flow, run (b), as plotted in Figure 10. The 

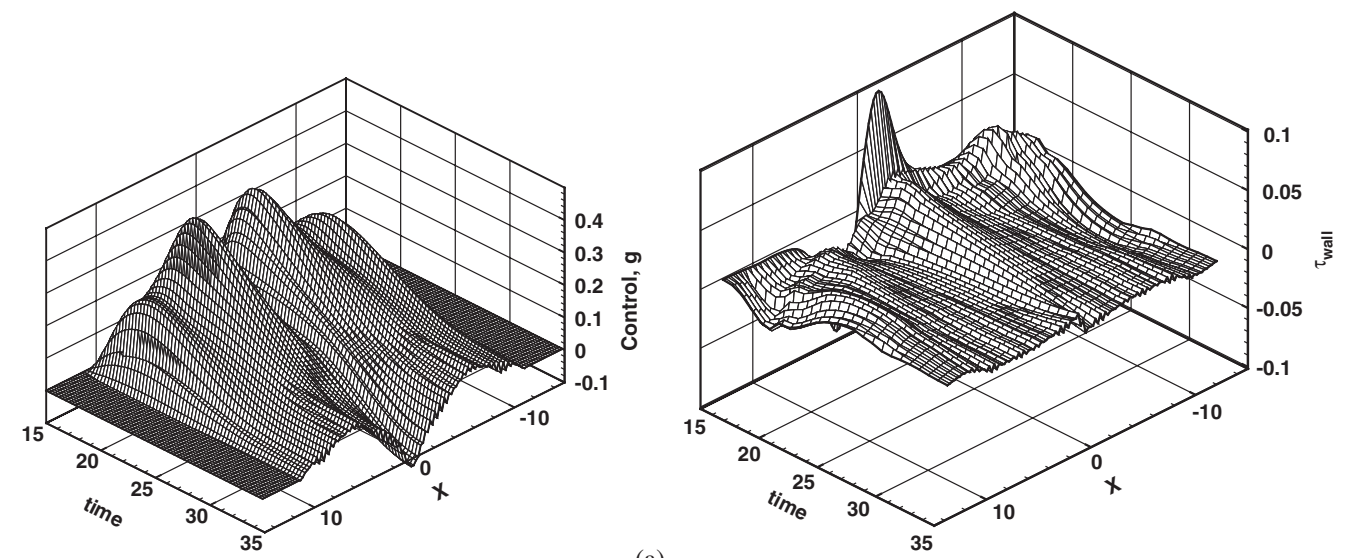

(a)
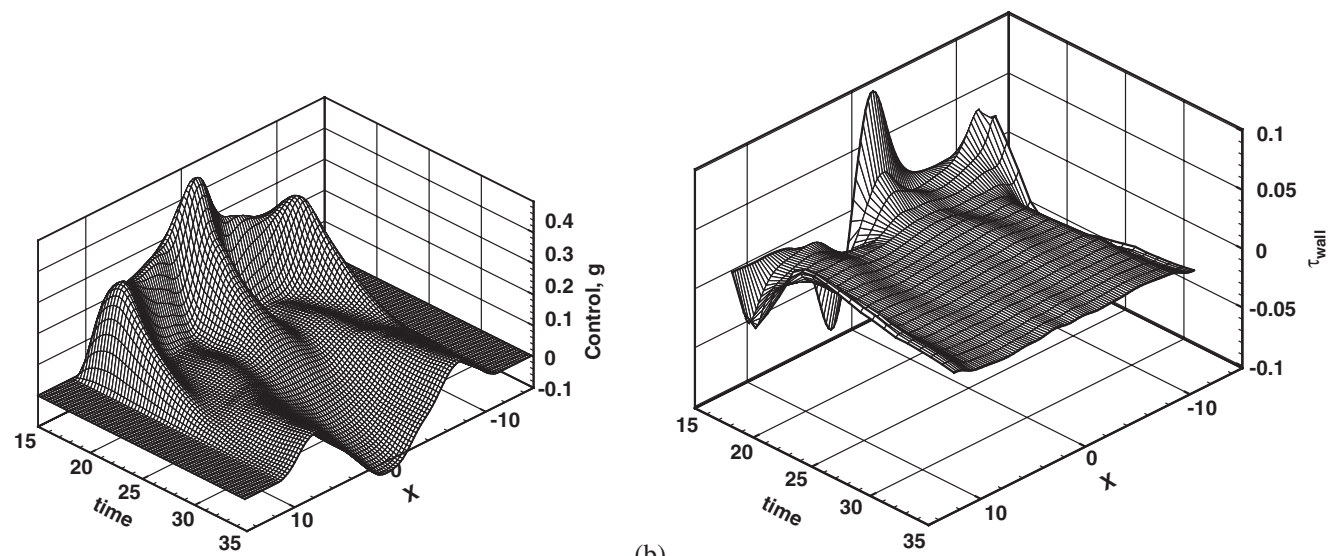

(b)

Figure 9. Control (left) and wall shear stress (right) distributions for optimal control runs (a) and (b): (a) $\alpha_{1}=\alpha_{2}=\alpha_{3}=0.5, \alpha_{4}=0$; and (b) $\alpha_{1}=\alpha_{2}=\alpha_{3}=0.5, \alpha_{4}=0.05$.

primary effect of the optimal control is to inject fluid at the wall that reduces the interaction between the vortices and the solid wall thereby reducing shear stress.

Table $\mathrm{V}$ itemizes values of the objective functional at the initial iterate $\left(J_{0}\right)$ and at the final control iterate $\left(J_{\text {final }}\right)$, as well as the observation term and individual regularization terms at the final control iterate. The reduction in the square magnitude of the shear-stree ranges from 26 times for (a) to 54 times for (b) compared to the uncontrolled flow.

\section{CONCLUSIONS}

We have studied the formulation of optimal boundary control for flows governed by the two-dimensional, unsteady, compressible Navier-Stokes equations including its impact on the numerical solution of these problems and we have presented numerical results illustrating the impact of problem formulation on three test problems. We have provided a careful 

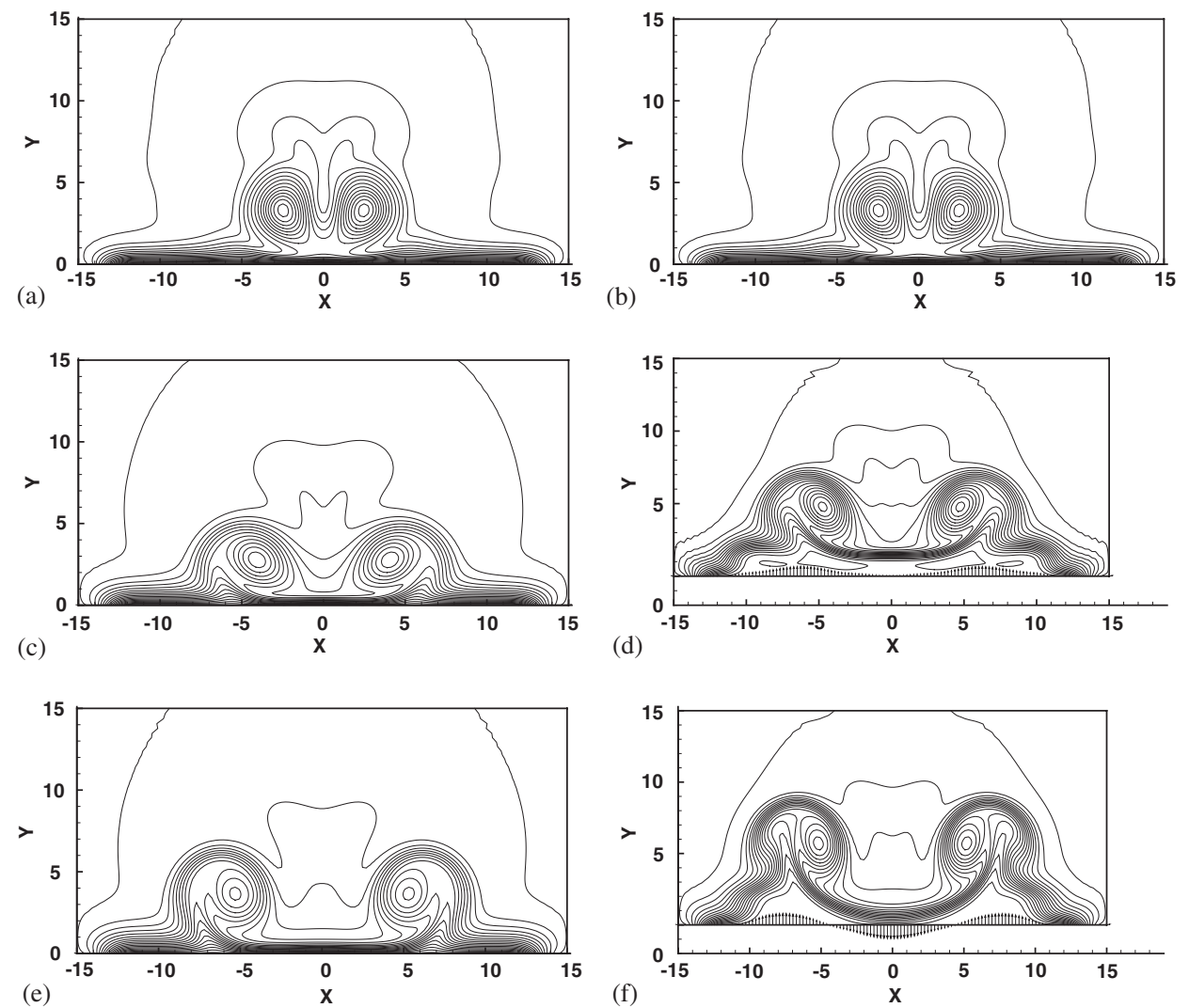

Figure 10. Density contours for the uncontrolled flow and drag control flow, run (b). There are 25 evenly spaced contours from 1.9 to 2.7. Arrows on the bottom wall in plots (d) and (f) represent the boundary control: (a) no control, $t=t_{0}$; (b) optimal control, run (b), $t=t_{0}$; (c) no control, $t=t_{0}+12.5$; (d) optimal control, run (b), $t=t_{0}+12.5$; (e) no control, $t=t_{f}$; and (f) optimal control, run (b), $t=t_{f}$.

Table V. Objective function values for wall shear stress control with different regularization parameters.

\begin{tabular}{lccccccc}
\hline Run & $J_{0}$ & $J_{\text {final }}$ & $\frac{1}{2} \int \omega \tau_{12}^{2}$ & $\alpha_{1} / 2 \int\left\|g_{t}\right\|_{2}^{2}$ & $\alpha_{2} / 2 \int\|g\|_{2}^{2}$ & $\alpha_{3} / 2 \int\|\nabla g\|_{2}^{2}$ & $\alpha_{4} / 2 \int\|\Delta g\|_{2}^{2}$ \\
\hline a & 222.88 & 11.225 & 8.546 & 0.187 & 2.124 & 0.367 & - \\
$\mathrm{b}$ & 222.88 & 7.455 & 4.148 & 1.075 & 1.956 & 0.230 & 0.05 \\
\hline
\end{tabular}

presentation of the gradient computation using the adjoint equation approach for the semidiscretized optimal control problem and the fully discretized optimal control problem. The gradient computation is strongly influenced by the selection of the control space, which is also related to the choice of the regularization term in the objective function. In many numerical approaches for the solution of optimal control problems governed by partial differential 
equations this dependence of the gradient computation on the choice of the control space is ignored which can lead to computed controls that exhibit grid dependent oscillations and degrade performance of the optimization algorithm. In our problems, only controls that have square integrable time and spatial derivatives are admissible. As a consequence, the gradient computation in the semi-discrete problem requires the solution of a very large-scale two-point boundary value problem. If higher-order spatial and time discretizations are used, this two-point boundary value is cumbersome and expensive to solve. As a remedy we have introduced an equivalent reformulation of the problem that avoids this task. This problem reformulation is also relevant for other applications. We have illustrated the impact of the problem formulation on the numerical solution using three test cases involving the control of wall-vortex interaction. All tests have shown that enforcing spatial and temporal regularity in the admissible controls is important to suppress artificial grid dependent oscillations in the computed controls. We have also observed a better performance of the non-linear conjugate gradient method if the control space is chosen appropriately and integrated into the numerical computations as described in this paper.

This paper describes the gradient computation for the semi-discretized and the fully discretized optimal control problems. This avoids the formulation of the adjoint differential equations and their boundary conditions. A formal derivation of the adjoint differential equations and their boundary conditions is given in Reference [21]. A more rigorous mathematical foundation of adjoint and gradient computations on the differential equation level is part of our future research. A comparison of discretizations of the adjoint differential equations and corresponding gradient expressions with the discrete adjoints and gradients computed as shown in this paper is under investigation. This work is a first step towards the on-blade control of aeroacoustic noise generated by blade-vortex interaction. Applications of the approach described in this paper to objective functions and geometries relevant for the control of aeroacoustic noise are under way [45].

\section{ACKNOWLEDGEMENTS}

The work of S.S. Collis, K. Ghayour and M. Heinkenschloss was supported by Texas ATP grant 003604-0001-1999, and in part by the Los Alamos National Laboratory Computer Science Institute (LACSI) through LANL contract number 03891-99-23, as part of the prime contract (W-7405-ENG36) between the Department of Energy and the Regents of the University of California. M. Ulbrich and S. Ulbrich were supported by DFG grants UL157/3-1 and UL158/2-1, respectively, and by CRPC grant CCR-9120008. Computations were performed on an SGI Origin 2000 which was purchased with the aid of NSF SCREMS grant 98-72009.

\section{REFERENCES}

1. Berggren M. Numerical solution of a flow-control problem: vorticity reduction by dynamic boundary action. SIAM Journal on Scientific Computing 1998; 19:829-860.

2. Bewley TR, Moin P, Temam R. DNS-based predictive control of turbulence: an optimal benchmark for feedback algorithms. Journal of Fluid Mechanics 2001; 447:179-225.

3. Collis SS, Chang Y. On the use of LES with a dynamic subgrid scale model for the optimal control of wall bounded turbulence. In Recent Advances in DNS and LES, Knight S, Sakell L (eds). Kluwer Academic Publishers: Dordrecht, 1999; 91-110.

4. Gunzburger MD, Manservisi S. The velocity tracking problem for Navier-Stokes flows with boundary control. SIAM Journal of Control Optimization 2000; 39:594-634. 
5. He H, Ghattas O, Antaki JF. Computational strategies for shape optimization of time-dependent NavierStokes flows. Technical Report CMU-CML-97-102, Engineering Design Research Center, Carnegie Mellon University, 1997. Electronically available from http://www.cs.cmu.edu/ oghattas/.

6. He J-W, Chevalier M, Glowinski R, Metcalfe R, Nordlander A, Periaux J. Drag reduction by active control for flow past cylinders. In Computational Mathematics Driven by Industrial Problems (Martina Franca, 1999). Burkard R, Deuflhard P, Jameson A, Lions JL, Strang G (eds.) Springer, Berlin, 2000; 287-363.

7. He J-W, Glowinski R, Metcalfe R, Nordlander A, Periaux J. Active control and drag optimization for flow past a circular cylinder. I. oscillatory cylinder rotation. Journal of Computational Physics 2000; 163:83-117.

8. He JW, Glowinski R, Metcalfe R, Periaux J. A numerical approach to the control and stabilization of advectiondiffusion systems: application to viscous drag reduction. International Journal of Computational Fluid Dynamics 1998; 11(1-2):131-156.

9. Homescu C, Navon M, Li Z. Supression of vortex shedding for flow around a cylinder using optimal control. International Journal for Numerical Methods in Fluids 2002; 38:43-69.

10. Joslin RD, Gunzburger MD, Nicolaides RA, Erlebacher G, Hussaini MY. Self-contained automated methodology for optimal flow control. AIAA Journal 1997; 35(5):816-824.

11. Li Z, Navon M, Hussaini MY, Le Dimet FX. Optimal control of cylinder wakes via suction and blowing. Computers and Fluids 2003; 32:149-171.

12. Borggaard J, Burns J. A PDE sensitivity equation method for optimal aerodynamic design. Journal of Computational Physics 1997; 136:366-384.

13. Cliff EM, Heinkenschloss M, Shenoy A. Airfoil design by an all-at-once method. International Journal for Computational Fluid Mechanics 1998; 11:3-25.

14. Jameson A, Martinelli L, Pierce NA. Optimum aerodynamic design using the Navier-Stokes equations. Theoretical and Computational Fluid Dynamics 1998; 10:213-237.

15. Jameson A, Reuther J. Control theory based airfoil design using the Euler equations. In AIAA Paper 94-4272, 1994.

16. Jou WH, Huffman WP, Young DP, Melvin RG, Bieterman MB, Hilems CL, Johnson FT. Practical considerations in aerodynamic design optimization. In Proceedings of the 12th AIAA Computational Fluid Dynamics Conference, San Diego, CA, June 19-22 1995, AIAA, AIAA Paper 95-1730, 950-960.

17. Soemarwoto B. The variational method for aerodynamic optimization using the Navier-Stokes equations. Technical Report 97-71, ICASE, NASA Langley Research Center, Hampton VA 23681-0001, 1997.

18. Gad el Hak M. (ed.). Flow Control, Passive, Active, and Reactive Flow Management. Cambridge University Press: New York, 2000.

19. Fursikov AV. Optimal Control of Distributed Systems. Theory and Applications. Translation of Mathematical Monographs, vol. 187. American Mathematical Society, Providence, RI, 2000.

20. Fursikov AV, Gunzburger MD, Hou LS. Boundary value problems and optimal boundary control for the NavierStokes systems: The two-dimensional case. SIAM Journal of Control Optimization 1998; 36:852-894.

21. Collis SS, Ghayour K, Heinkenschloss M, Ulbrich M, Ulbrich S. Numerical solution of optimal control problems governed by the compressible Navier-Stokes equations. In Optimal Control of Complex Structures, International Series of Numerical Mathematics, Hoffmann KH, Lasiecka I, Leugering G, Sprekels J, Tröltzsch F (eds.) vol. 139. Birkhäuser: Basel, 2001; 43-55.

22. Collis SS, Ghayour K, Heinkenschloss M, Ulbrich M, Ulbrich S. Towards adjoint-based methods for aeroacoustic control. In 39th Aerospace Science Meeting \& Exhibit, 2001. Electronically available from http://www.caam.rice.edu/heinken.

23. Hoff D. Discontinuous solutions of the Navier-Stokes equations for multidimensional flows of heat-conducting fluids. Archive for Rational Mechanics and Analysis 1997; 139:303-354.

24. Lions PL. Mathematical Topics in Fluid Mechanics. Volume 2, Compressible Models, Oxford Lecture Series in Mathematics and its Applications, vol. 10. Claredon Press: Oxford, 1998.

25. Matsumura A, Nishida T. The initial value problem for the equations of motion of viscous and heat-conductive gases. Journal of Mathematics of Kyoto University 1980; 20:67-104.

26. Valli A. Mathematical results for compressible flows. In Mathematical Topics in Fluid Mechanics, Pitman Research Notes Mathematics, Rodriguez JF, Sequeira A (eds), vol. 274. Longman Scientific and Technical: New York, Essex, 1992.

27. Dautray R, Lions JL. Mathematical Analysis and Numerical Methods for Science and Technology, Functional and Variational Methods, vol. 2. Springer: Berlin, Heidelberg, New York, 1988

28. Dautray R, Lions JL. Mathematical Analysis and Numerical Methods for Science and Technology, Evolution Problems I, vol. 5. Springer: Berlin, Heidelberg, New York, 1992.

29. Renardy M, Rogers RC. An Introduction to Partial Differential Equations. Texts in Applied Mathematics, vol. 13. Springer: Berlin, Heidelberg, New York, 1993.

30. Lele SK. Compact finite difference schemes with spectral-like resolution. Journal of Computational Physics 1992; 103(1):16-42.

31. Tam CKW, Webb JC. Dispersion-relation-preserving finite difference schemes for computational acoustics. Journal of Computational Physics 1993; 107:262-281. 
32. Tam CKW, Webb JC, Dong Zhong. A study of the short wave components in computational acoustics. Journal of Computational Acoustics 1993; 1(1):1-30.

33. Collis SS. A computational investigation of receptivity in high-speed-flow near a swept leading-edge. Ph.D. Thesis. Standford University, 1997. Electronically available from $\mathrm{http}: / /$ mems.rice.edu/ collis

34. Carpenter MH, Gottlieb D, Abarbanel S. Stable and accurate boundary treatments for compact, high-order finite difference schemes. Applied Numerical Mathematics 1993; 12(1-3):55-87.

35. Dennis Jr. JE, Schnabel RB. Numerical Methods for Nonlinear Equations and Unconstrained Optimization. SIAM: Philadelphia, 1996.

36. Heinkenschloss M, Vicente LN. An interface between optimization and application for the numerical solution of optimal control problems. ACM Transactions on Mathematical Software 1999; 25:157-190.

37. Hager WW. Runge-Kutta methods in optimal control and the transformed adjoint system. Numerische Mathematik 2000; 87:247-282.

38. Lui G, Nocedal J, Waltz R. Cg+: conjugate gradient software. http://www.ece.nwu.edu/ nocedal/software.html.

39. Nocedal J, Wright SJ. Numerical Optimization. Springer: Berlin, Heidelberg, New York, 1999.

40. Ulbrich M. Constrained optimal control of Navier-Stokes flow by semi-smooth Newton methods. Systems \& Control Letters, 2002, at press.

41. Restrepo JM, Leaf GK, Griewank A. Circumventing storage limitations in variational data assimilation studies. SIAM Journal of Scientific Computing 1998; 19:1586-1605.

42. Giering R, Kaminski T. Recipes for adjoint code construction. ACM Transactions on Mathematical Software 1998; 24:437-474.

43. Colonius T, Lele SK, Moin P. The free compressible viscous vortex. Journal of Fluid Mechanics 1991; 230: $45-73$.

44. Alekseev AK, Navon IM. The analysis of an ill-posed problem using multi-scale resolution and second-order adjoint techniques. Computer Methods in Applied Mechanics and Engineering 2000; 190:1937-1953.

45. Collis SS, Ghayour K, Heinkenschloss M. Optimal control of aeroacoustic noise generated by cylinder vortex interaction. International Journal of Aeroacoustics 2002; 1:97-114. 